\title{
Effect of an oblique magnetic field on the superparamagnetic relaxation time. II. Influence of the gyromagnetic term
}

\author{
W. T. Coffey* \\ Department of Electronic and Electrical Engineering, Trinity College, Dublin 2, Ireland \\ D. S. F. Crothers \\ Department of Applied Mathematics and Theoretical Physics, The Queen's University of Belfast, Belfast BT7, 1NN, \\ Northern Ireland \\ J. L. Dormann ${ }^{\dagger}$ \\ Laboratoire de Magnétisme et d'Optique de l'Université de Versailles, UKA 151 Bâtiment Fermat, \\ 45 Avenue des États Unis, 78035, Versailles, Cedex, France \\ L. J. Geoghegan and E. C. Kennedy \\ Department of Applied Mathematics and Theoretical Physics, The Queen's University of Belfast, Belfast BT7, 1NN, \\ Northern Ireland
}

(Received 23 May 1997; revised manuscript received 29 December 1997)

\begin{abstract}
The effect of a uniform magnetic field applied at an oblique angle to the easy axis of magnetization on the superparamagnetic (longitudinal or Néel) relaxation time is investigated by numerically solving the FokkerPlanck equation for the smallest nonvanishing eigenvalue. It is demonstrated that the reciprocal of the asymptotic formula for the Kramers escape rate in the intermediate to high damping limit for general nonaxially symmetric potentials when applied to the present problem, yields an acceptable asymptotic approximation to the Néel time for moderate to high values of the damping. Alternatively the corresponding Kramers low dissipation formula (energy controlled diffusion) provides an acceptable approximation for very small values of the damping. The effect of the gyromagnetic term which gives rise to coupling between the longitudinal and transverse modes of motion generally corresponds to an increase of the smallest nonvanishing eigenvalue and so to a decrease of the Néel relaxation time. The integral relaxation time or area under the slope of the curve of the decay of the magnetization is also evaluated. It is demonstrated that for sufficiently high values of the uniform field (much less, however, than that required to destroy the bistable nature of the potential) the reciprocal of the lowest nonvanishing eigenvalue (proportional to the Néel time, or the time of reversal of the magnetization) and the integral relaxation time may differ exponentially from one another signifying the contributions of modes other than that associated with the overbarrier (Néel) relaxation process to the overall relaxation process. The overall behavior is qualitatively similar (apart from the azimuthal dependence) to that of the axially symmetric case which arises due to the depletion of the shallower of the two potential wells by the uniform field, so that the fast processes in the deeper of the two wells may come to dominate the relaxation process at sufficiently high values of the uniform field. [S0163-1829(98)04229-5]
\end{abstract}

\section{INTRODUCTION}

The calculation of the relaxation time $\tau$ of the magnetic moment of a single-domain ferromagnetic particle under the influence of an applied field is very important because the value of $\tau$ obtained provides a comprehensive description of the experimental results. For example, in the most common experiment which is used for characterizing particle assemblies, i.e., the zero field cooled magnetization $M_{\mathrm{ZFC}}$, the temperature of the $M_{\mathrm{ZFC}}$ maximum can be determined by comparing $\tau$ with the inverse of the heating rate, ${ }^{1}$ so that an analytic expression for $\tau$ is required for a quantitative determination of the parameters of the relaxing system. Now in actual particle assemblies, the easy axes are in random directions with respect to the field orientation, therefore the calculation of $\tau$ must be performed for a field at an oblique angle $\psi$ to the direction of the easy axis (the original calculations of the relaxation time by Brown and Aharoni $^{2-4}$ were restricted to the case of axial symmetry which requires that the field and easy axis should be colinear). Such a calculation may be directly checked by comparing it with experimental results on either a particle assembly or on particle arrays or on isolated particles (monoparticle sample). These are the only systems for which samples are available at present. ${ }^{5-8}$ Furthermore, the experimental and theoretical $\tau$ values may also be used to determine the contribution of other rotation modes. In essence, the Néel-Brown model ${ }^{9,10}$ on which the calculation of $\tau$ is based assumes a single-domain particle and a synchronous (coherent) rotation of the spins. However, depending on the size, shape, and parameters of the particle, other rotation modes (incoherent magnetization processes) may be more favorable energetically. Evidence of such has been provided by the authors of Ref. 5, although their measurements were performed on relatively large and irregularly shaped samples. More recently, Wernsdorfer et al. (see Ref. 7) have presented some very interesting results by comparing 
their experimental observations to the predictions of the curling mode of magnetization reversal in an infinite cylinder, where the exchange length $d_{0}$ defined as $d_{0}=2 \sqrt{A} / M_{s}(A$ is the exchange constant and $M_{s}$ is the mean magnetization of a nonrelaxing particle) defines the transition from uniform rotation to curling. They have shown that for nanowires of diameter smaller than $100 \mathrm{~nm}$, the process of uniform nucleation is valid, whereas for wires having a diameter larger than twice the exchange length $d_{0}$, nucleation occurs at several almost degenerate fields at values close to the curling instability. For wires whose diameters are close to the exchange length, the Stoner-Wohlfarth model may be used, where the switching time and field measurements indicate that a single energy barrier is dominant and the reversal process may be described by an Arrhenius law. ${ }^{7}$ However, when considering samples which are small when compared with the exchange length (which is the topic of discussion in this paper), recent experiments (see Ref. 8) have shown that the concept of uniform rotation is valid in these cases. Although analytical considerations indicate that synchronous rotation (as is assumed in the Néel-Brown model) is not in principle possible for a nonellipsoidal particle, nevertheless further experiments and calculations ${ }^{11}$ for $\mathrm{NiFe}_{2} \mathrm{O}_{4}$ particles demonstrate that magnetic disordering occurs at the particle surface due to the lack of interactions. Thus only small perturbations to synchronous rotations may take place. Our calculations could bring some of these issues into discussion by checking whether or not an approach based on the synchronous rotation assumption actually works.

A preliminary discussion of the effect of an external uniform magnetic field, applied at an oblique angle $\psi$ to the easy axis of magnetization of a single-domain ferromagnetic particle, on the Néel or superparamagnetic relaxation time for uniaxial anisotropy has recently ${ }^{12}$ been published by us. The calculation of the Néel relaxation time is in general accomplished by determining the smallest nonvanishing eigenvalue $\lambda_{1}$ of the appropriate Fokker-Planck equation. $\lambda_{1}$ is the rate constant associated with the longest surviving decay mode (Néel mode) of the magnetization. The reciprocal of $\lambda_{1}$ in the high barrier limit is approximately ${ }^{3}$ the time of reversal of the magnetization (that is, the mean first passage time, Néel time, or time taken to climb the potential hill) over the potential barrier due to the crystalline anisotropy and the applied field. We have presented ${ }^{12}$ both an exact numerical solution for $\lambda_{1}$ and an asymptotic estimate based on an extension ${ }^{3,12,13}$ of the Kramers theory ${ }^{14}$ of the thermally activated escape of particles over potential barriers to include spin relaxation. The numerical calculations we have hitherto presented, however, all proceed from the assumption that the dimensionless damping parameter $a$ is so large that the effect of the gyromagnetic (precessional) term (which contains $1 / a$ as a prefactor) on the Néel time may be ignored. Thus the results pertain only to the very high damping limit and so are quite restricted.

The omission of the gyromagnetic terms has the advantage that the set of differential recurrence relations for the aftereffect functions of the magnetization to which the Fokker-Planck equation for the distribution function of the magnetization orientations may be reduced by expanding its solution as a series of spherical harmonics $X_{l, m}(\theta, \varphi)$ in the polar angles $\theta$ and $\varphi$ (specifying the orientation of the mag- netic moment) reduces to a nine term rather than a thirteen term one between the numbers $l$ and $m$. Furthermore the recurrence relations for negative $m$ may easily be expressed in terms of those for positive $m$ by means of the symmetry properties of the $X_{l, m}$ [because the expectation values of the spherical harmonics $x_{l, m}(t)$ are real when the gyromagnetic terms are ignored] so greatly reducing the amount of computer time required for the solution. If the gyromagnetic terms are included, the $x_{l, m}(t)$ are complex. It is then impossible to express the recurrence relation for the $x_{l,-m}(t)$ in terms of that of the $x_{l, m}(t)$, only in terms of that of the $x_{l, m}^{*}(t)$ (the star denotes the complex conjugate). Hence the recurrence relations may only be solved by expressing the solution of the Fokker-Planck equation in terms of surface spherical harmonics. These lead to recurrence relations for the real and imaginary parts of the $x_{l, m}(t)$ as we shall describe later. As far as the asymptotic estimate of $\lambda_{1}$ is concerned the omission of the gyromagnetic term indicated at first that an axially symmetric approximation ${ }^{12,15,16}$ might be made in the Kramers approach to the problem so reducing it to an effective one variable problem as only the reaction rate coordinate $\theta$ (colatitude) will now be involved. This, however, leads to asymptotic estimates which deviate appreciably from the numerical solution so that a more rigorous treatment including both reaction coordinates $\theta, \varphi$ must be given.

Our neglect of the gyromagnetic term besides having the obvious disadvantage (when the treatment is extended to calculate the complex magnetic susceptibility) that ferromagnetic resonance phenomena, ${ }^{17}$ etc., may not be included implies that the calculation of $\lambda_{1}$ will be invalid when the damping parameter $a$ is finite. This is important in view of the very recent experimental results of Dormann et al. ${ }^{18}$ on $\gamma$ $\mathrm{Fe}_{2} \mathrm{O}_{3}$ with weak interparticle interactions which indicate that $a$ may be as small as 0.05 , hence the influence of $a$ on $\lambda_{1}$ will be substantial.

Here the gyromagnetic effects are included in the calculation of $\lambda_{1}$ and hence the Néel relaxation time so that the calculation is valid for all values of the damping parameter. Both an exact solution for $\lambda_{1}$ based on the thirteen term differential recurrence relations arising from the FokkerPlanck equation including the gyromagnetic term and a rigorously derived estimate of $\lambda_{1}$ based on a two variable Kramers approach as used for a general nonaxially symmetric potential by Brown ${ }^{10}$ and Smith and de Rozario ${ }^{19}$ (in a discussion of relaxation in cubic anisotropy potentials) which allows one to include the influence of the gyromagnetic term in the prefactor of such an asymptotic estimate will be presented. The two variable ${ }^{12,14}$ asymptotic estimate is essentially a modification of the Kramers ${ }^{14,20}$ intermediate to high damping (IHD) limit calculation of the escape rate for a particle diffusing in phase space. In addition, the value of $\lambda_{1}$ for very small values of the damping parameter $a$ will be compared with the very low damping (LD) limit, using the formalism of Ref. 21 , of the Kramers ${ }^{14}$ theory as adapted to the magnetic problem by Klik and Gunther. ${ }^{22}$ This allows one ${ }^{3}$ to state the range of values of the damping parameter $a$ for which a particular formula of Kramers ${ }^{14}$ is valid. Furthermore the integral relaxation time $\tau$ which is the area under the curve of the decay of the magnetization following a change in the applied field will be calculated as far as terms 


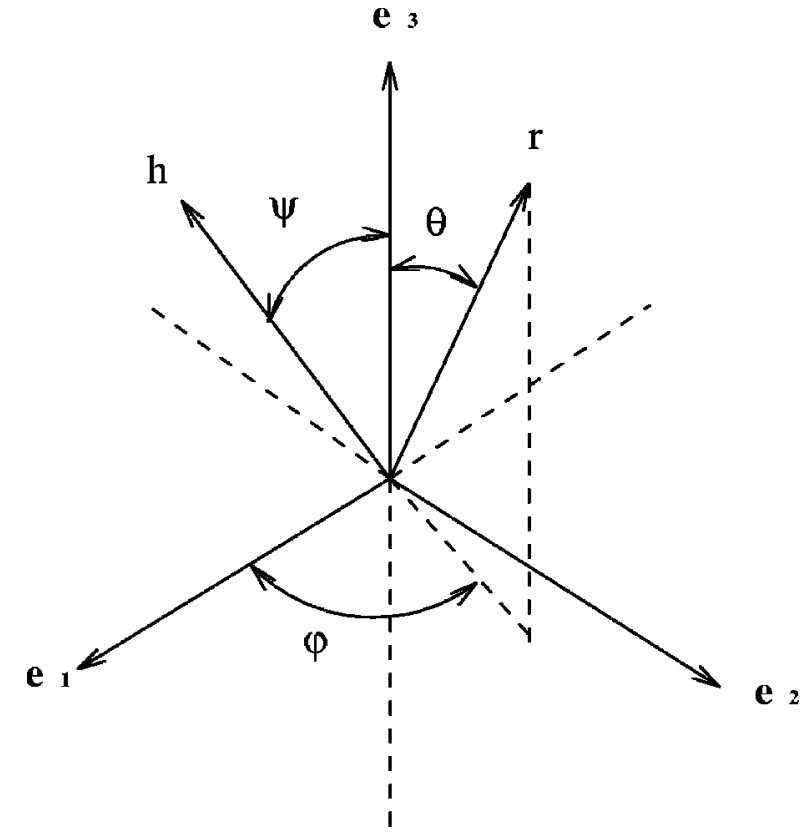

FIG. 1. External field and magnetization orientations in terms of spherical polar coordinates.

linear in the perturbation, which means that the integral relaxation time may be identified with the correlation time of the autocorrelation function of the magnetization.

\section{MAGNETIC RESPONSE FUNCTIONS AND CHARACTERISTIC TIMES}

In the particular nonaxially symmetric problem of uniaxial anisotropy with the applied field at an angle $\psi$ to the easy axis which we are considering, the (Gibbs) free energy density per unit volume is

$$
V(\mathbf{r})=\beta^{-1} \sigma\left[1-\left(\mathbf{r} \cdot \mathbf{e}_{\mathbf{3}}\right)^{2}\right]-\beta^{-1} \xi(\mathbf{r} \cdot \mathbf{h}),
$$

where

$$
\mathbf{r}=\mathbf{M} / M_{s} .
$$

$M_{s}$ is the mean magnetization of a nonrelaxing particle and the polar angles $\theta$ and $\varphi$ (see Fig. 1) specify the orientation of $\mathbf{M}$.

$$
\sigma=K v / k T
$$

is the anisotropy parameter,

$$
\xi=H M_{s} v / k T
$$

is the external field parameter, and $\mathbf{h}$ is the orientation of the external magnetic field $\mathbf{H}$. The orthogonal coordinate system $\mathbf{e}_{1}, \mathbf{e}_{2}, \mathbf{e}_{3}$ is chosen so that $\mathbf{e}_{3}$ is parallel to the anisotropy axis and $\mathbf{h}$ is parallel to the plane containing $\mathbf{e}_{1}$ and $\mathbf{e}_{3}$ (see Fig. 1), hence

$$
\mathbf{h}=\sin \psi \mathbf{e}_{1}+\cos \psi \mathbf{e}_{3} .
$$

The distribution of orientations $W(\mathbf{r}, t)$ of the magnetization $\mathbf{M}$ of an assembly of noninteracting single-domain ferromagnetic particles on the unit sphere satisfies the Fokker-Planck equation $^{10,12}$

$$
\dot{W}=L_{\mathrm{FP}} W
$$

where $^{10,12}$

$$
\begin{aligned}
L_{\mathrm{FP}} W= & \beta^{-1} \nabla^{2} W+b \nabla^{2} V+b\left(\frac{\partial V}{\partial \theta} \frac{\partial W}{\partial \theta}+\frac{1}{\sin ^{2} \theta} \frac{\partial V}{\partial \varphi} \frac{\partial W}{\partial \varphi}\right) \\
& +\frac{b}{a \sin \theta}\left(\frac{\partial V}{\partial \theta} \frac{\partial W}{\partial \varphi}-\frac{\partial V}{\partial \varphi} \frac{\partial W}{\partial \theta}\right) .
\end{aligned}
$$

The operator $\nabla^{2}$ denotes the angular part of the Laplacian, that is

$$
\begin{gathered}
\nabla^{2}=\frac{1}{\sin \theta} \frac{\partial}{\partial \theta}\left(\sin \theta \frac{\partial}{\partial \theta}\right)+\frac{1}{\sin ^{2} \theta} \frac{\partial^{2}}{\partial \varphi^{2}}, \\
\beta=v / k T,
\end{gathered}
$$

where $v$ is the volume of the magnetic particle and $k T$ is the thermal energy,

$$
b=\frac{a \gamma}{\left(1+a^{2}\right) M_{s}},
$$

and the diffusional relaxation time is

$$
\tau_{N}=\beta / 2 b
$$

(We remark that $b$ is often denoted in the literature by $h^{\prime}$ while $b / a$ is denoted by $g^{\prime}$.) Here $a=\eta \gamma M_{s}$ (also denoted by $\alpha$ ) is the dimensionless damping parameter, $\eta$ is the phenomenological damping constant from Gilbert's equation, ${ }^{13,22,23}$ and $\gamma$ is the gyromagnetic ratio. The first three terms on the right hand side of Eq. (7) are purely dissipative terms and the last term prefixed by $b / a$ is the gyromagnetic term. In Ref. $12 a$ is assumed to be so large that the influence of the gyromagnetic term on the Néel relaxation time $\tau$, where

$$
\tau=\frac{2 \tau_{N}}{\lambda_{1}}=\frac{(\beta / b)}{\lambda_{1}}
$$

is negligible. $\lambda_{1}$ is the smallest nonvanishing eigenvalue of $-(\beta / b) L_{\mathrm{FP}}$.

In order to calculate $\lambda_{1}$ including the effects of the gyromagnetic term we shall first calculate the linear response of the system following a small decrease $\mathbf{H}_{1}$ in the field $\mathbf{H} . \mathbf{H}_{1}$ is such that

$$
v M_{s} H_{1} / k T=\xi_{1} \ll 1
$$

so that terms $O\left(\xi_{1}^{2}\right)$ may be ignored. Thus the initial condition for linear response may be imposed by replacing $\xi$ with $\xi+\xi_{1}$ for $t \leqslant 0$ where $\xi_{1} \ll \xi$. The system is assumed to be in thermodynamic equilibrium at $t=0, \infty$, so that both the initial and final distributions are of Maxwell-Boltzmann type, namely,

$$
W(\mathbf{r}, 0)=\frac{e^{-\beta V+\xi_{1} \mathbf{r} \cdot \mathbf{h}}}{\int e^{-\beta V+\xi_{1} \mathbf{r} \cdot \mathbf{h}} d \Omega},
$$




$$
W(\mathbf{r}, \infty)=\frac{e^{-\beta V}}{\int e^{-\beta V} d \Omega} .
$$

Here the volume element is $d \Omega=\sin \theta d \theta d \varphi$ and the surface integrals are over the unit sphere. The relaxation behavior is described by the response function

$$
f(t)=\lim _{\xi_{1} \rightarrow 0} \xi_{1}^{-1}\left(\langle\mathbf{r} \cdot \mathbf{h}\rangle-\langle\mathbf{r} \cdot \mathbf{h}\rangle_{0}\right)=\sum_{i} A_{i} e^{-b \beta^{-1} \lambda_{i} t}, \quad t>0,
$$

where $\lambda_{1}, \lambda_{2}, \lambda_{3}, \ldots ; \quad\left\{0<\left|\lambda_{1}\right|<\left|\lambda_{2}\right|<\left|\lambda_{3}\right|<\ldots\right\}$ are the eigenvalues and $A_{1}, A_{2}, A_{3}, \ldots$, the corresponding amplitudes of $-(\beta / b) L_{\mathrm{FP}}$ and $^{13,15}$

$$
\begin{aligned}
\langle\mathbf{r} \cdot \mathbf{h}\rangle & =\int \mathbf{r} \cdot \mathbf{h} W(\theta, \varphi, t) d \Omega \\
& =\int(\cos \theta \cos \psi+\sin \theta \cos \varphi \sin \psi) W d \Omega
\end{aligned}
$$

represents the decay of the average cosine in the direction of the field. The subscript zero denotes that the statistical average is to be evaluated in the absence of the perturbation $\xi_{1}$.

The normalized complex susceptibility $\chi(\omega)$ may now be evaluated since according ${ }^{13}$ to linear response theory $\chi(\omega)$ is given by

$$
\begin{aligned}
\chi(\omega) / \chi^{\prime}(0) & =1-i \omega \int_{0}^{\infty} e^{-i \omega t} f(t) d t \\
& =\sum_{k} \frac{A_{k}}{1+i \omega \beta /\left(\lambda_{k} b\right)} / \sum_{k} A_{k} .
\end{aligned}
$$

A global quantity associated ${ }^{3}$ with the decay of the magnetization in addition to the mean first passage time (which is the lifetime of the longest decay mode) is the integral relaxation time (which is the area under the curve of the decay of the magnetization, i.e., the correlation time, since the response is linear). The integral relaxation time is given by ${ }^{3,24}$

$$
T_{c}=b \beta^{-1} \int_{0}^{\infty} f(t) d t / f(0)=\sum_{k} \lambda_{k}^{-1} A_{k} / \sum_{k} A_{k} .
$$

The integral relaxation time, because it is the area under the curve of the decay of the magnetization, is a relaxation time which provides a measure of the relative contributions of all the decay modes of the system (both long and short lived). In measurements of the reversal of the magnetization, however, we are concerned only with the longest lived (Néel) mode. In high frequency measurements such as those of the complex susceptibility, the integral relaxation time rather than the Néel time should be used, ${ }^{3}$ since at high frequencies, the contribution of the fast modes is significant. In order to calculate the smallest nonvanishing eigenvalue (which is essentially the reciprocal of the mean first passage time), it is first necessary (as in Ref. 12) to represent the Fokker-Planck equation in Eq. (6) as a differential recurrence relation which is then converted to a first order matrix differential equation with constant coefficients. The smallest nonvanishing eigen- value is then the smallest root of the characteristic equation of the system where a set of equations large enough to ensure convergence of the system is taken. This may be accomplished by expanding the distribution function in spherical harmonics as we shall now describe.

\section{MATRIX FORMULATION OF THE FOKKER-PLANCK EQUATION}

The nonaxially symmetric nature of the Gibbs free energy means that the distribution function in the Fokker-Planck equation is also nonaxially symmetric. The smallest nonvanishing eigenvalue of the Fokker-Planck equation could in principle be calculated by posing the calculation of $\lambda_{1}$ as a Sturm-Liouville problem. A better method in this case, however, when two variables are involved is that one should expand the solution of the Fokker-Planck equation as a series of spherical harmonics (Fourier-Laplace series). This leads to a set of differential recurrence relations for the Fourier coefficients.

The spherical harmonics are defined by ${ }^{25}$

$$
X_{l, m}(\theta, \varphi) \equiv P_{l}^{m}(\cos \theta) e^{i m \varphi}, \quad|m| \leqslant l,
$$

where $P_{m}^{l}$ denotes the associated Legendre functions. In addition we shall require the normalized spherical harmonics $N_{l, m} X_{l, m}$, where the normalization factor is given by

$$
N_{l, m}=(-1)^{m} \sqrt{\frac{(2 l+1)(l-m) !}{4 \pi(l+m) !}} .
$$

We remark that the normalized spherical harmonics form a complete biorthonormal basis for $L^{2}\{\Omega\}, \Omega=\{\mathbf{r},\|\mathbf{r}\|=1\}$ (the Hilbert space of square integrable functions which are defined on the unit sphere). We further remark that the spherical harmonics $X_{l, m}$ satisfy $^{25}$ the symmetry relation (the star denotes complex conjugate)

$$
X_{l,-m}=\rho_{l, m} X_{l, m}^{*}, \quad \rho_{l, m}=(-1)^{m} \frac{(l-m) !}{(l+m) !} .
$$

We shall now suppose that the solution of Eq. (6) has a representation as a Fourier series

$$
W(\mathbf{r}, t)=\sum_{|m| \leqslant l} N_{l, m}^{2} x_{l, m}^{*}(t) X_{l, m}(\mathbf{r}) .
$$

Now Eq. (1) becomes

$$
\begin{gathered}
\beta V=\sigma\left(1-\alpha_{3}^{2}\right)-\xi\left(\alpha_{1} \sin \psi+\alpha_{3} \cos \psi\right), \\
\beta \nabla^{2} V=-2 \sigma\left(1-3 \alpha_{3}^{2}\right)+2 \xi\left(\alpha_{1} \sin \psi+\alpha_{3} \cos \psi\right),
\end{gathered}
$$

where the $\alpha_{i}=\mathbf{r e}_{i}$, that is,

$$
\alpha_{1}=\sin \theta \cos \varphi, \quad \alpha_{2}=\sin \theta \sin \varphi, \quad \alpha_{3}=\cos \theta
$$

are the direction cosines of the magnetization. In addition the Fokker-Planck operator when it acts on a spherical harmonic (that is $L_{\mathrm{FP}} X_{l, m}$ ) has the following representation [Eq. (2.113) of Ref. 15 and Eq. (39) of Ref. 21] 


$$
\begin{aligned}
L_{\mathrm{FP}} X_{l, m}= & b\left[-l(l+1) \beta^{-1}+\frac{l(l+1)}{2} V+\frac{1}{2} \nabla^{2} V\right] X_{l, m} \\
& +\frac{i b(l+m)(l-m+1)}{2 a}\left(\frac{\partial V}{\partial \alpha_{1}}+i \frac{\partial V}{\partial \alpha_{2}}\right) X_{l, m-1} \\
& -i b a^{-1} m \frac{\partial V}{\partial \alpha_{3}} X_{l, m}+\frac{i b}{2 a}\left(\frac{\partial V}{\partial \alpha_{1}}-i \frac{\partial V}{\partial \alpha_{2}}\right) X_{l, m+1} .
\end{aligned}
$$

Thus utilizing the recurrence relations of the spherical harmonics as in Ref. 15 we then find that ${ }^{15,21}$ the expectation values $x_{l, m}$ of the spherical harmonics $X_{l, m}$ satisfy the differential recurrence relations [Eq. (3.28) and Eq. (3.29) of Ref. 15]

$$
\dot{x}_{l, m}=\left(X_{l, m} \dot{W}\right)=\left(X_{l, m} L_{\mathrm{FP}} W\right)=b \beta^{-1} \sum_{p, q} e_{l, m, p, q} x_{p, q}
$$

(the braces denote the inner product). The $x_{l,-m}$ may be written in terms of the $x_{l, m}^{*}$ by means of the symmetry relation

$$
x_{l,-m}=\rho_{l, m} x_{l, m}^{*}
$$

which follows immediately from Eq. (22). Explicit expressions for the matrix elements $e_{l, m, p, q}$ are given in Appendix A. Equation (27) constitutes a set of differential recurrence relations for the expectation values $x_{l, m}$ of the spherical harmonics $X_{l, m}$. These will allow us to write down the differential recurrence relations for the aftereffect function $f(t)$ in Eq. (15). In order to achieve this, we introduce the surface spherical harmonics $^{25}$ (the introduction of these avoids a complex coefficient matrix hence greatly reducing the amount of computer time required), namely,

$$
\begin{gathered}
U_{l, 0}=X_{l, 0}, \quad U_{l, m}=(1 / \sqrt{2})\left(X_{l, m}+X_{l, m}^{*}\right)=\sqrt{2} \operatorname{Re} X_{l, m}, \\
U_{l,-m}=(1 / i \sqrt{2})\left(X_{l, m}-X_{l, m}^{*}\right)=\sqrt{2} \operatorname{Im} X_{l, m}, \quad 0<m \leqslant l,
\end{gathered}
$$

and form differential recurrence relations in terms of these remarking that the normalized surface spherical harmonics $N_{l,|m|} X_{l,|m|}$ also form a complete biorthonormal basis for $L^{2}\{\Omega\}$. Thus we suppose that the solution of Eq. (6) has a representation given by

$$
W(\mathbf{r}, t)=\sum_{|m| \leqslant l} N_{l,|m|}^{2} u_{l, m}(t) U_{l, m}(\mathbf{r})
$$

then the expectation values $u_{l, m}$ of the surface harmonics $U_{l, m}$ satisfy another set of differential-recurrence relations, namely,

$$
\dot{u}_{l, m}=\left(\dot{W}, U_{l, m}\right)=\left(L_{\mathrm{FP}} W, U_{l, m}\right)=b \beta^{-1} \sum_{p, q} a_{l, m, p, q} u_{p, q} .
$$

The new matrix elements of which are given by

$$
a_{l, m, p, q}=\beta b^{-1} N_{p,|q|}^{2}\left(U_{l, m}, L_{\mathrm{FP}} U_{p, q}\right) .
$$

These may easily be related to the matrix elements $e_{l, m, p, q}$ defined by Eq. (27) as follows. For $m, q>0$ we form the matrices

$$
\begin{gathered}
A_{l, m, p, q}=\left(\begin{array}{cc}
a_{l, m, p, q} & a_{l, m, p,-q} \\
a_{l,-m, p, q} & a_{l,-m, p,-q}
\end{array}\right), \\
A_{l, m, p, 0}=\left(\begin{array}{c}
a_{l, m, p, 0} \\
a_{l,-m, p, 0}
\end{array}\right), \quad R_{l, m}=\rho_{l, m}\left(\begin{array}{cc}
1 & 0 \\
0 & -1
\end{array}\right), \\
A_{l, 0, p, q}=\left(\begin{array}{ll}
a_{l, 0, p, q} & a_{l, 0, p,-q}
\end{array}\right), \\
A_{l, 0, p, 0}=a_{l, 0, p, 0}, \quad R_{l, 0}=1
\end{gathered}
$$

and construct the following matrices from the matrix elements $e_{l, m, p, q}$ :

$$
\begin{gathered}
E_{l, m, p, q}=\left(\begin{array}{cc}
\operatorname{Re} e_{l, m, p, q} & -\operatorname{Im} e_{l, m, p, q} \\
\operatorname{Im} e_{l, m, p, q} & \operatorname{Re} e_{l, m, p, q}
\end{array}\right), \\
E_{l, m, p, 0}=\sqrt{2}\left(\begin{array}{c}
\operatorname{Re} e_{l, m, p, 0} \\
\operatorname{Im} e_{l, m, p, 0}
\end{array}\right), \\
E_{l, 0, p, q}=(1 / \sqrt{2})\left(\operatorname{Re} e_{l, 0, p, q}-\operatorname{Im} e_{l, 0, p, q}\right), \\
E_{l, 0, p, 0}=\operatorname{Re} e_{l, 0, p, 0} .
\end{gathered}
$$

The various matrices above are related to one another by means of the equation

$$
A_{l, m, p, q}=E_{l, m, p, q}+E_{l, m, p,-q} R_{p, q} .
$$

The matrix representation of the differential operator $(\beta / b) L_{\mathrm{FP}}$ is now

$$
A=\left[\begin{array}{cccc}
A_{1,0,1,0} & A_{1,0,1,1} & A_{1,0,2,0} & \cdots \\
A_{1,1,1,0} & A_{1,1,1,1} & A_{1,1,2,0} & \cdots \\
A_{2,0,1,0} & A_{2,0,1,1} & A_{2,0,2,0} & \cdots \\
\vdots & \vdots & \vdots & \vdots
\end{array}\right] .
$$

This is now suitable for numerical computation of the response function as will be described below.

\section{RECURRENCE RELATIONS FOR THE RESPONSE FUNCTION AND THE INITIAL CONDITIONS}

In order to set up the equations for the calculation of the response function from Eqs. (31)-(36) we introduce the column vectors

$$
\begin{aligned}
& U=\left(u_{1,0}, \quad u_{1,1}, \quad u_{1,-1}, \quad u_{2,0}, \quad u_{2,1}, \ldots\right)^{T} \text {, } \\
& B=(2 \xi \cos \psi / 3, \quad 2 \sqrt{2} \xi \sin \psi / 3, \\
& 0, \quad 4 \sigma / 5, \quad 0, \quad 0, \quad \ldots)^{T} \text {, }
\end{aligned}
$$

where $U$ satisfies the set of simultaneous differential equations

$$
\dot{U}=A U+B \text {. }
$$


The final equilibrium condition after removal of the perturbing field $\mathbf{H}_{1}$ (i.e., a small step decrease in the magnitude of the external uniform magnetic field) is given by

$$
\dot{U}(\infty)=0
$$

so that the final equilibrium values vector

$$
U_{\infty}=U(\infty)
$$

is found by solving ${ }^{13}$ the set of simultaneous linear equations

$$
A U_{\infty}=-B .
$$

The decay modes ${ }^{12}$ of the magnetization, namely,

$$
C=\lim _{\xi_{1} \rightarrow 0}\left(U-U_{\infty}\right),
$$

expressed in matrix form satisfy the set of simultaneous differential equations

$$
\dot{C}=A C \text {. }
$$

Let $c_{l, m}$ denote the components of $C$. It then follows from Eqs. (14) and (15) that the initial values (that is just before the removal of the initial perturbing field) are given by

$$
\begin{aligned}
c_{l, m}(0)= & \lim _{\xi_{1} \rightarrow 0} \xi_{1}^{-1}\left[\frac{\int U_{l, m} e^{-\beta V+\xi_{1} \mathbf{r} \cdot \mathbf{h}} d \Omega}{\int e^{-\beta V+\xi_{1} \mathbf{r} \cdot \mathbf{h}} d \Omega}\right. \\
& \left.-\frac{\int U_{l, m} e^{-\beta V} d \Omega}{\int e^{-\beta V} d \Omega}\right] \\
= & \frac{\int \mathbf{r} \cdot \mathbf{h} U_{l, m} e^{-\beta V} d \Omega}{\int e^{-\beta V} d \Omega} \\
& -\frac{\int \mathbf{r} \cdot \mathbf{h} e^{-\beta V} d \Omega \int U_{l, m} e^{-\beta V} d \Omega}{\int e^{-\beta V} d \Omega} \frac{\int e^{-\beta V} d \Omega}{\int} .
\end{aligned}
$$

This may be written in a more compact form

$$
\begin{aligned}
c_{l, m}(0) & =\left(\partial u_{l, m} / \partial \xi\right)(\infty)=\left\langle\mathbf{r} \cdot \mathbf{h} U_{l, m}\right\rangle_{0}-\langle\mathbf{r} \cdot \mathbf{h}\rangle_{0}\left\langle U_{l, m}\right\rangle_{0} \\
& =\sum_{p, q} b_{l, m, p, q} u_{p, q}(\infty)
\end{aligned}
$$

Let $w_{l, m, p, q}$ denote the coefficients when the $u_{l, m}$ are replaced by the $x_{l, m}$ then the $w_{l, m, p, q}$ are found by applying the spherical harmonic recurrence relations and are given in Appendix B. The matrix elements $b_{l, m, p, q}$ may then be determined from the $w_{l, m, p, q}$ using the transformation defined in Eq. (35) ( $a_{l, m, p, q}$ and $e_{l, m, p, q}$ replaced by $b_{l, m, p, q}$ and $w_{l, m, p, q}$, respectively). The principle is the same as that used for the axially symmetric case. ${ }^{13}$ The initial values vector is then given by

$$
C_{0}=C(0)=W U_{\infty}+\left(\begin{array}{llll}
\frac{1}{3} \cos \psi, & \frac{\sqrt{2}}{3} \sin \psi, & 0, & \ldots
\end{array}\right)^{T} .
$$

The zero frequency Laplace transforms vector is determined from Eq. (44) by solving the simultaneous equations

$$
A \widetilde{C}(0)=C(0) .
$$

The solution of Eq. (44) is given by

$$
C(t)=S e^{b \beta^{-1} L t} K,
$$

where $L$ is the diagonal matrix whose components are the eigenvalues $\lambda_{1}, \lambda_{2}, \lambda_{3}, \ldots$, and

$$
S=\left(\begin{array}{ccc}
S_{1}^{1,0} & S_{2}^{1,0} & \ldots \\
S_{1}^{1,1} & S_{2}^{1,1} & \ldots \\
\vdots & \vdots & \vdots
\end{array}\right)
$$

is the matrix whose column vectors are the eigenvectors of $A$. The vector

$$
K=\left(\begin{array}{llll}
k_{1}, & \left.k_{2}, \quad \ldots, \quad k_{N}\right)^{T}
\end{array}\right.
$$

is found by solving the set of simultaneous linear equations

$$
S K=C_{0} .
$$

The response function in Eq. (15) may be determined from the decay modes because

$$
f(t)=\cos \psi c_{1,0}(t)+\sin \psi c_{1,1}(t)=\sum_{i} A_{i} e^{-b \beta^{-1} \lambda_{i} t},
$$

so that the amplitudes defined by Eq. (18) are given by

$$
A_{i}=k_{i}\left[\cos \psi S_{i}^{1,0}+(1 / \sqrt{2}) \sin \psi S_{i}^{1,1}\right]
$$

and the expression for the correlation time in Eq. (18) becomes

$$
T_{c}=\frac{\cos \psi \tilde{c}_{1,0}(0)+\sin \psi \tilde{c}_{1,1}(0)}{\cos \psi c_{1,0}(0)+\sin \psi c_{1,1}(0)} .
$$

We now have formal expressions for all the quantities required to describe the response following a small perturbation in the external field, namely, the response function $f(t)$ in Eq. (15), the complex susceptibility $\chi(\omega)$ in Eq. (17), the correlation time $T_{c}$ in Eq. (18), and the mean first passage time or Néel time ${ }^{3} \tau=2 \tau_{N} / \lambda_{1}$. Both numerical and analytic aspects of the problem will be greatly simplified if we have an approximate analytic expression for $\lambda_{1}$ in the high barrier limit. Such an expression serves as an important check on the validity of the numerical calculations while simultaneously providing an analytic formula ${ }^{3}$ which may be compared with experiment. The calculation of such an asymptotic formula is much more difficult in a nonaxially symmetric problem involving two reaction coordinates such as the present one, than in an axially symmetric problem where the mean first passage time depends only on a single reaction coordinate (namely, on the latitude) and exact analytic solutions for $\tau$ for every barrier height exist. ${ }^{3}$ Nevertheless the intermediate 
to high damping (IHD) method of Kramers ${ }^{14,20}$ (as generalized by Langer and Hänggi et $a l^{26,27}$ ) for the treatment of the escape of particles (diffusing in phase space so that the process is described by two variables, namely, position and velocity) over potential barriers in the IHD barrier limit may be applied to the present problem. This two variable Kramers procedure (although not mentioned explicitly as such by them) appears to have been first introduced into the theory of superparamagnetism (without reference to Langer's ${ }^{26}$ work) by Smith and de Rozario ${ }^{19}$ in a discussion of relaxation in cubic anisotropy potentials, it was in effect also used by Eisenstein and Aharoni ${ }^{28}$ and generalized to an arbitrary nonaxially symmetric potential by Brown ${ }^{10}$ and later discussed by Klik and Gunther ${ }^{22}$ in relation to the Kramers ${ }^{14}$ two variable theory. In the following section we shall apply the two variable IHD method to the calculation of asymptotic expressions for $\lambda_{1}$ for the problem in hand. We shall also provide an asymptotic formula for $\lambda_{1}$ in the very low dissipation limit following the Klik-Gunther ${ }^{22}$ adaptation of the uniform expansion of the mean first passage time method of Matkowsky, Schuss, and $\operatorname{Tier}^{29}$ to the general nonaxially symmetric superparamagnetic problem.

\section{USE OF BROWNS HIGH-ENERGY BARRIER APPROXIMATION FOR NONAXIALLY SYMMETRIC POTENTIALS}

We have shown in detail ${ }^{12}$ for the potential of Eq. (23) if the parameter ${ }^{15} h=\xi / 2 \sigma$ satisfies the condition

$$
h<h_{c},
$$

where

$$
\begin{aligned}
h_{c} & =\left[(\sin \psi)^{2 / 3}+(\cos \psi)^{2 / 3}\right]^{-3 / 2} \\
& =\sqrt{1+\tan ^{2} \psi}\left[1+(\tan \psi)^{2 / 3}\right]^{-3 / 2}, \quad 0 \leqslant \psi \leqslant \pi / 2
\end{aligned}
$$

then the free energy $V$ [of Eq. (1)] remains bistable having a maximum $V_{0}$, say, separated by minima $V_{1}, V_{2}$. The smallest nonvanishing eigenvalue $\lambda_{1}$ of the Fokker-Planck equation, Eq. (6), is the rate of escape of magnetic moments over the potential barrier characterized by the maximum $V_{0}$. The condition in Eq. (56) was originally given by Stoner and Wohlfarth ${ }^{30}$ in their discussion of the construction of hysteresis loops from the potential given by Eq. (1) and by Pfeiffer ${ }^{31}$ who studied the reversal of the magnetization (the present problem) in the discrete orientation ${ }^{9}$ (Néel) approximation. We have already derived ${ }^{12}$ by neglecting the gyromagnetic term in Eq. (7) and imposing axial symmetry, an approximate asymptotic formula for $\lambda_{1}$ in the limit of high potential barriers. This formula is Eq. (72) of Ref. 12, namely,

$$
\begin{aligned}
\lambda_{1}= & \frac{1}{2 \pi} \sin \theta_{0} \sqrt{-\beta V^{\prime \prime}\left(\theta_{0}\right)}\left\{\frac{\sqrt{\beta V^{\prime \prime}\left(\theta_{1}\right)}}{\sin \theta_{1}} e^{-\beta\left[V\left(\theta_{0}\right)-V\left(\theta_{1}\right)\right]}\right. \\
& \left.+\frac{\sqrt{\beta V^{\prime \prime}\left(\theta_{2}\right)}}{\sin \theta_{2}} e^{-\beta\left[V\left(\theta_{0}\right)-V\left(\theta_{2}\right)\right]}\right\} .
\end{aligned}
$$

This equation is simply Brown's original ${ }^{2}$ (1963) calculation for an axially symmetric potential which has minima at $\theta$ $=0, \pi$ when applied to the case where the potential has minima at $\theta=\theta_{1}, \theta_{2}$ where $0 \leqslant \theta \leqslant \pi$; it is related, although in essence ${ }^{3,22}$ entirely different in origin, to the translational single reaction coordinate problem considered by Kramers. ${ }^{14}$ Such an axially symmetric calculation leads to a rough estimate of $\lambda_{1}$ in the high barrier limit as described in Ref. 12, however, the application of it to a nonaxially symmetric potential cannot be justified. The two reaction coordinates $\theta$ and $\varphi$ must be included in the calculation of the asymptotic estimate by adapting the Kramers calculation ${ }^{14,20}$ of the escape rate of a particle moving in phase space in the IHD limit to the present problem (essentially using the approach of Lange ${ }^{26}$ ). As we have mentioned this was achieved by Brown ${ }^{10}$ (without reference to Langer, and without any discussion of the range of values of the damping parameter for which it is valid ) by calculating the flow of representative points across a saddle point of the potential (that is a point where the potential is a minimum with respect to the direction cosine $\alpha_{1}$ and a maximum with respect to the direction cosine $\alpha_{2}$ ). The result holds good for any well behaved nonaxially symmetric potential, as far as the present problem is concerned (for details see Refs. 10 and 15).

The IHD calculation of Brown ${ }^{10}$ supposes that the free energy per unit volume $V=V(\mathbf{r})$ has a bistable structure with minima at $\theta_{1}$ and $\theta_{2}$ separated by a potential barrier that contains a saddle point at $\theta_{0}$ (where it is assumed that the $\theta_{i}$ are coplanar). If one denotes the plane containing $\theta_{i}$ by $\Pi$, then for each $i=0,1,2$, one can define an orthogonal triad of unit vectors $E_{i}=\left(e_{1}^{(i)}, e_{2}^{(i)}, e_{3}^{(i)}\right)$ with $e_{1}^{(i)} \perp \Pi$ and $e_{2}^{(i)}, e_{3}^{(i)}$ $\in \Pi$. If

$$
X_{i}^{T}=\left(\alpha_{1}^{(i)}, \alpha_{2}^{(i)}, \alpha_{3}^{(i)}\right)
$$

denotes the coordinate vectors (direction cosines) of $\mathbf{r}$ with respect to $E_{i}$, respectively, and $\mathbf{r}$ is close to the stationary point $\theta_{i}$ of the potential, then $\mathbf{r}=E_{i} X_{i}$ and $V(\mathbf{r})$ can be approximated to the second order of small quantities by the Taylor series

$$
V=V_{i}+\frac{1}{2}\left[c_{1}^{(i)}\left(\alpha_{1}^{(i)}\right)^{2}+c_{2}^{(i)}\left(\alpha_{2}^{(i)}\right)^{2}\right] .
$$

In the context of the present problem we introduce the parameters

$$
u=h \cos \psi, \quad \nu=h \sin \psi,
$$

then the condition for a stationary point of the Gibbs free energy is

$$
(x+u) \sqrt{1-x^{2}} \pm \nu x=0,
$$

where $x=\cos \theta$. This is Eq. (28) of Ref. 12 where the negative sign corresponds to the stationary points which occur for $\varphi=0$ while the positive sign for the local maximum which occurs for $\varphi=\pi$. The truncated Taylor expansion in Eq. (59) yields (after a long calculation which has been discussed at length in Ref. 15)

$$
\begin{gathered}
V_{i}=\beta^{-1} \sigma\left(1-x_{i}^{2}-2 u x_{i}-2 \nu \sqrt{1-x_{i}^{2}}\right), \\
c_{1}^{(i)}=2 \beta^{-1} \sigma\left(x_{i}^{2}+u x_{i}+\nu \sqrt{1-x_{i}^{2}}\right), \\
c_{2}^{(i)}=2 \beta^{-1} \sigma\left(-1+2 x_{i}^{2}+u x_{i}+\nu \sqrt{1-x_{i}^{2}}\right),
\end{gathered}
$$


where $-1 \leqslant x_{2} \leqslant x_{0} \leqslant x_{0}^{\prime} \leqslant x_{1} \leqslant 1$ are the roots of the quartic equation

$$
(x+u)^{2}\left(1-x^{2}\right)=\nu^{2} x^{2}
$$

which is obtained by squaring Eq. (61).

We shall now suppose that the ratios of barrier height to thermal energy become appreciable, i.e., $\beta\left(V_{0}-V_{i}\right) \gg 1$, so that we may assume that the density of magnetic moment orientations $W$ rapidly achieves a state of quasiequilibrium ${ }^{10}$ thus the Fokker-Planck equation in Eq. (6) reduces to the master equation

$$
\dot{n}_{1}=-\dot{n}_{2}=\nu_{2,1} n_{2}-\nu_{1,2} n_{1}
$$

and

$$
\lambda_{1} \approx \beta b^{-1}\left(\nu_{1,2}+\nu_{2,1}\right) .
$$

Thus after a very lengthy calculation which is analogous to that of Langer ${ }^{26}$ and is detailed fully in Ref. 15 , Sec. V we have essentially Brown's asymptotic IHD formula, Eq. (84) of Ref. 10 or Eq. (5.60) of Ref. 15, namely,

$$
\begin{aligned}
\lambda_{1} \equiv & \beta\left(\sqrt{c_{1}^{(1)} c_{2}^{(1)}} e^{-\beta\left(V_{0}-V_{1}\right)}+\sqrt{c_{1}^{(2)} c_{2}^{(2)}} e^{-\beta\left(V_{0}-V_{2}\right)}\right) \\
& \times \frac{-c_{1}^{(0)}-c_{2}^{(0)}+\sqrt{\left(c_{2}^{(0)}-c_{1}^{(0)}\right)^{2}-4 a^{-2} c_{1}^{(0)} c_{2}^{(0)}}}{4 \pi \sqrt{-c_{1}^{(0)} c_{2}^{(0)}}} .
\end{aligned}
$$

$\lambda_{1}$ may be evaluated analytically in three distinct cases. First $\psi=0$, the axially symmetric case. Here the process depends on the single reaction coordinate $\theta$ (and so is valid for all values of the damping parameter) and we have the axially symmetric results of Brown ${ }^{2}$ and Aharoni ${ }^{4}$ (an exact integral solution for the problem may be obtained from the theory of mean first passage times as described in Ref. 3) and asymptotically,

$$
\lambda_{1}=\frac{2 \sigma^{3 / 2}}{\sqrt{\pi}}\left(1-h^{2}\right)\left[(1+h) e^{-\sigma(1+h)^{2}}+(1-h) e^{-\sigma(1-h)^{2}}\right] .
$$

The other cases are $\psi=\pi / 2, \pi / 4$ which were also considered by Pfeiffer $^{31}$ in the discrete orientation approximation. Here the quartic equation (65) may be easily factorized. For $\psi=\pi / 2$, Eq. (65) becomes

$$
x^{2}\left(1-h^{2}-x^{2}\right)=0,
$$

hence

$$
x_{2}=-\sqrt{1-h^{2}}, \quad x_{0}=0, \quad x_{1}=\sqrt{1-h^{2}} .
$$

Equations (62)-(64) give

$$
\begin{gathered}
\beta\left(V_{0}-V_{i}\right)=\sigma(1-h)^{2}, \quad i=1,2, \quad c_{1}^{(1)}=c_{1}^{(2)}=2 K, \\
c_{2}^{(1)}=c_{2}^{(2)}=2 K\left(1-h^{2}\right), \quad c_{1}^{(0)}=2 K h, \\
c_{2}^{(0)}=-2 K(1-h),
\end{gathered}
$$

hence

$$
\lambda_{1}=\frac{\sigma\left[1-2 h+\sqrt{1+4 a^{-2} h(1-h)}\right] \sqrt{(1+h)}}{\pi \sqrt{h}} e^{-\sigma(1-h)^{2}} .
$$

Thus the $\nu_{i, j}$ of the two level system described by Eq. (67) are degenerate in this case. We remark that the axially symmetric approximation Eq. (82) of Ref. 12 differs from Eq. (73) in the high damping limit $a \rightarrow \infty$, simply by the factor $\sqrt{h}$ so that both formulas become asymptotic to each other as $h \rightarrow 1$. In the other soluble case $\psi=\pi / 4$, the stationary condition Eq. (61) becomes

$$
x \sqrt{1-x^{2}}+\frac{h}{\sqrt{2}}\left(\sqrt{1-x^{2}}-x\right)=0 .
$$

Let

$$
w=\sqrt{1-x^{2}}-x,
$$

then Eqs. (74) and (75) yield, respectively, the pair of simultaneous quadratic equations

$$
\begin{gathered}
2 x^{2}+2 w x+\left(w^{2}-1\right)=0, \\
w^{2}-h \sqrt{2} w-1=0 .
\end{gathered}
$$

The roots of Eq. (77) are

$$
w_{ \pm 1}=\frac{h \pm \sqrt{h^{2}+2}}{\sqrt{2}},
$$

hence the simultaneous solutions of Eqs. (76) and (77) are the four roots of the pair of quadratic equations obtained on replacing $w$ in Eq. (76) with $w_{ \pm 1}$ in Eq. (78), i.e.,

$$
\begin{aligned}
x_{ \pm 1} & =\frac{w_{1}}{2} \pm \frac{1}{\sqrt{2}} \sqrt{1-\frac{w_{1}^{2}}{2}} \\
& =-\frac{h+\sqrt{h^{2}+2}}{2 \sqrt{2}} \pm \frac{\sqrt{1-h^{2}+h \sqrt{h^{2}+2}}}{2},
\end{aligned}
$$

$$
\begin{aligned}
x_{ \pm 1}^{\prime} & =-\frac{w-1}{2} \pm \frac{1}{\sqrt{2}} \sqrt{1-\frac{w_{-1}^{2}}{2}} \\
& =\frac{-h-\sqrt{h^{2}+2}}{2 \sqrt{2}} \pm \frac{\sqrt{1-h^{2}+h \sqrt{h^{2}+2}}}{2} .
\end{aligned}
$$

Let

$$
S_{ \pm 1}=\sqrt{\frac{1-h^{2} \pm h \sqrt{h^{2}+2}}{2}},
$$

then Eqs. (62)-(64) yield for the various constants in Eq. (68)

$$
\begin{aligned}
& \beta\left(V_{0}-V_{1}\right)=\frac{\sigma}{2} \sqrt{h^{2}+2}\left(S_{1}+S_{-1}\right), \\
& \beta\left(V_{0}-V_{2}\right)=\sigma S_{-1}\left(\sqrt{h^{2}+2}-3 h\right),
\end{aligned}
$$




$$
\begin{gathered}
c_{1}^{(1)}=\beta^{-1} \sigma\left[1+\left(h+\sqrt{h^{2}+2}\right) S_{1}\right], \\
c_{2}^{(1)}=2 \beta^{-1} \sigma S_{1} \sqrt{h^{2}+2}, \\
c_{1}^{(0)}=\beta^{-1} \sigma\left[1+\left(h-\sqrt{h^{2}+2}\right) S_{-1}\right], \\
c_{2}^{(0)}=-2 \beta^{-1} \sigma S_{-1} \sqrt{h^{2}+2}, \\
c_{1}^{(2)}=\beta^{-1} \sigma\left[1+\left(-h+\sqrt{h^{2}+2}\right) S_{-1}\right], \\
c_{2}^{(2)}=2 \beta^{-1} \sigma S_{-1} \sqrt{h^{2}+2} .
\end{gathered}
$$

which are explicit forms for the constants in Eq. (68). As far as the other values of $\psi$ are concerned, the application of the algebraic formula for the roots of the quartic equation (65) so as to express $\lambda_{1}$ of Eq. (68) in terms of $u$ and $\nu$ is not recommended due to the proximity of the roots at $x_{0}$ and $x_{0}^{\prime}$. For accurate results a numerical method such as NewtonRaphson should be used instead. The asymptotic estimates for $\lambda_{1}$ which we have presented above are based on the IHD case of the Kramers theory. The original investigations by Smith and de Rozario ${ }^{19}$ and Brown ${ }^{10}$ did not state ${ }^{3}$ the range of values of the friction for which the IHD formula Eq. (68) was valid. This problem was first addressed by Klik and Gunther $^{22}$ who presented a formula for $\tau^{-1}=2 \tau_{n} / \lambda_{1}$ while considering for convenience one escape path only (that over the lower barrier). The asymptotic equation in their 1990 paper was given as

$$
\tau^{-1}=\frac{a \gamma}{\pi M_{s}} \sqrt{c_{1}^{(2)} c_{2}^{(2)}}\left(V_{0}-V_{2}\right) e^{-\beta\left(V_{0}-V_{2}\right)}
$$

Equation (83) is similar to the low damping formula of Kramers, ${ }^{14}$ however, Kramers ${ }^{14}$ included a factor of $1 / 2$ in his calculations. This factor must also be included ${ }^{3}$ in the formula of Klik and Gunther, ${ }^{22}$ and so we obtain ${ }^{3,32}$

$$
\tau^{-1}=\frac{a \gamma}{2 \pi M_{s}} \sqrt{c_{1}^{(2)} c_{2}^{(2)}}\left(V_{0}-V_{2}\right) e^{-\beta\left(V_{0}-V_{2}\right)} .
$$

If we extend Eq. (84) to include both escape paths, it becomes

$$
\begin{aligned}
\tau^{-1}= & \frac{a \gamma}{2 \pi M_{s}}\left[\sqrt{c_{1}^{(2)} c_{2}^{(2)}}\left(V_{0}-V_{2}\right) e^{-\beta\left(V_{0}-V_{2}\right)}\right. \\
& \left.+\sqrt{c_{1}^{(1)} c_{2}^{(1)}}\left(V_{0}-V_{1}\right) e^{-\beta\left(V_{0}-V_{1}\right)}\right] .
\end{aligned}
$$

Using the fact that

$$
b=\frac{a \gamma}{\left(1+a^{2}\right) M_{s}} \approx \frac{a \gamma}{M_{s}}
$$

for small $a$, we can form an expression for $\lambda_{1}$,

$$
\begin{aligned}
\lambda_{1}= & \frac{\beta}{2 \pi}\left[\sqrt{c_{1}^{(2)} c_{2}^{(2)}}\left(V_{0}-V_{2}\right) e^{-\beta\left(V_{0}-V_{2}\right)}\right. \\
& \left.+\sqrt{c_{1}^{(1)} c_{2}^{(1)}}\left(V_{0}-V_{1}\right) e^{-\beta\left(V_{0}-V_{1}\right)}\right] .
\end{aligned}
$$

Hence, the low damping results for $\lambda_{1}$ are given by Eq. (87). Klik and Gunther ${ }^{22}$ introduced a criterion for the validity of the IHD formula and the low damping formula in that the
Plot of $\lambda$ versus $\sigma, h=0.1,0.2, a=0.2$

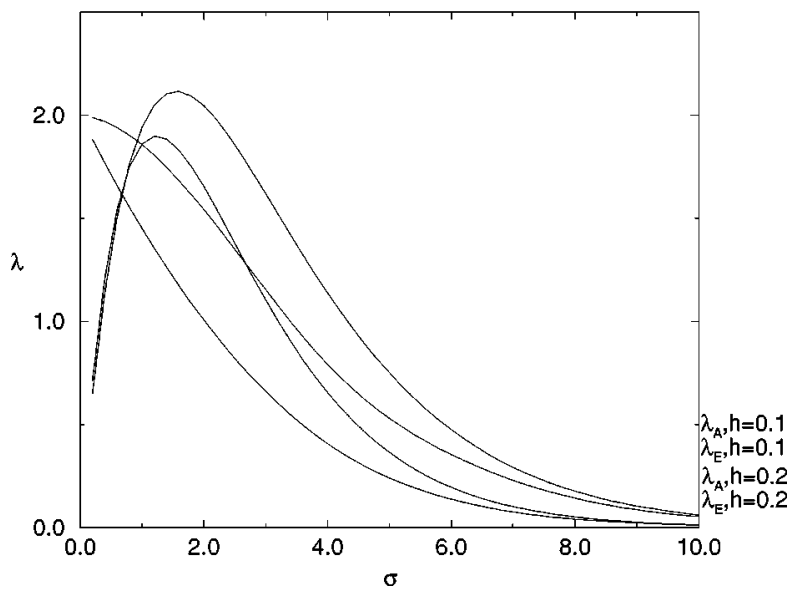

FIG. 2. Comparison of exact $\left(\lambda_{1}=\lambda_{E}\right)$ and asymptotic $\left(\lambda_{1}\right.$ $=\lambda_{A}$ ) expressions including the gyromagnetic term. Evidently, Eq. (73) yields a good approximation to the high barrier solution in both cases.

IHD formula is applied in cases where $a \beta\left(V_{0}-V_{2}\right)$ $\gg 1, \beta\left(V_{0}-V_{2}\right)>1$, and the low damping formula of Eq. $(87)$ is used in cases where $a \beta\left(V_{0}-V_{2}\right) \ll 1, \beta\left(V_{0}-V_{2}\right)>1$. A detailed discussion of this is provided in Ref. 3 .

\section{IMPLEMENTATION OF NUMERICAL COMPUTATIONS}

Exact values of $\lambda_{1}$ were computed by programming the matrix form of the Fokker-Planck equation [as described by Eq. (36)] in FORTRAN, where the indices $l, m$ of the matrix elements $A_{l, m, p, q}$ in Eq. (35) are used to determine the row positioning of the elements, and the indices $p, q$ are used to determine the column positioning of the elements. The lowest eigenvalue $\lambda_{1}$ is determined by diagonalizing the matrix using an eispack routine RGG. To ensure convergence for each set of parameters $h, \sigma, a, \psi$, the matrix size parameter lmax [where the order of the square matrix is given by $\operatorname{lmax} *(\operatorname{lmax}+2)]$ is increased repeatedly until successive values of $\lambda_{1}$ lie within a tolerance of $10^{-4}$. By using this procedure, we can ensure accuracy of the results.

The barrier height parameter $\sigma$ dominates the order of the matrix required in order to obtain convergence. For example, when the barrier height parameter is increased from $\sigma=5$ to $\sigma=10$, the matrix size parameter lmax is increased to ensure convergence, and generally, CPU time requirements for $\sigma$ $=10$ are more than ten times that of the cases where $\sigma=5$. It has been observed in the calculation of $\lambda_{1}$, that the diagonal terms of the matrices involved increase as $n^{2}$, where $n$ is the order of the square matrices. This would explain the increasing difficulty of the problem, and the increase in CPU time requirements as the dimensions of the matrices increase.

\section{NUMERICAL EVALUATION OF THE NÉEL TIME AND COMPARISON WITH ASYMPTOTIC ESTIMATES}

In this section we compare our numerical calculations of the Néel time as yielded by the formula 
Plot of $\lambda_{A} / \lambda_{B}$ versus $\sigma$ for $h=0.1,0.2,0.4,0.5,0.7,0.8$

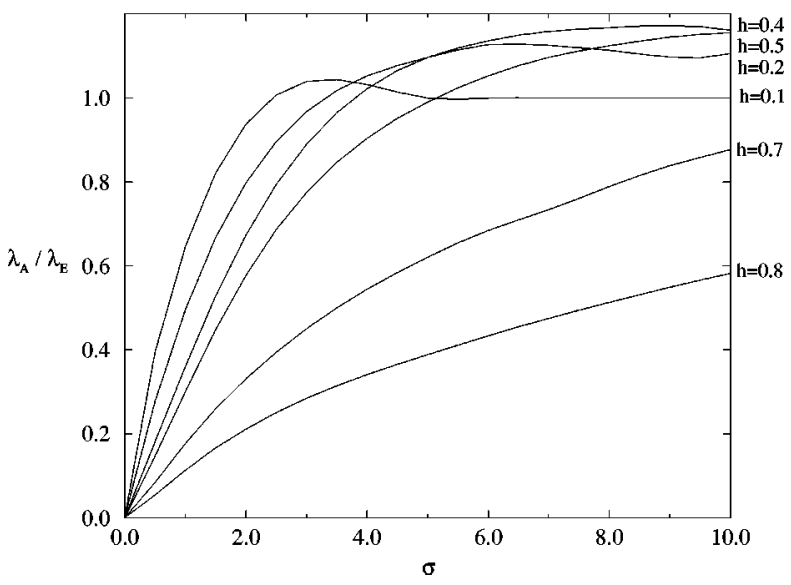

FIG. 3. Comparison of the solution $\lambda_{1}(\pi / 2)$ yielded by the asymptotic formula [Eq. (73)] in the high damping limit $a \rightarrow \infty$ with the exact numerical solution. It is apparent that the systematic error in Fig. 13 of Ref. 11 is removed by the correct asymptotic formula.

$$
\tau=2 \tau_{N} / \lambda_{1}
$$

with the asymptotic estimates for $\psi=\pi / 2, \pi / 4$ yielded by Eqs. (68), (73) and Eqs. (68), (74)-(82) which are the only cases where explicit solution of the quartic equation (65) is possible. Thus in Fig. 2 (for $\psi=\pi / 2$ ) we show the behavior of $\lambda_{E}$ which is $\lambda_{1}$ obtained from the exact numerical solution when compared with $\lambda_{A}$ which is the behavior yielded by the nonaxially symmetric asymptotic formula Eq. (68) $(\psi=\pi / 2)$ [which leads to Eq. (73)] for a value of the dimensionless damping parameter $a=0.2$. It appears from these figures that the asymptotic formula Eq. (68) provides a good approximation to $\lambda_{E}$ for large barrier heights. This is reinforced by the results shown in Fig. 3 which are for infinite $a$ values (the high damping limit). It is apparent that the ratio

Plot of $\lambda$ versus $\psi a=0.1, h=0.1, \sigma=10$

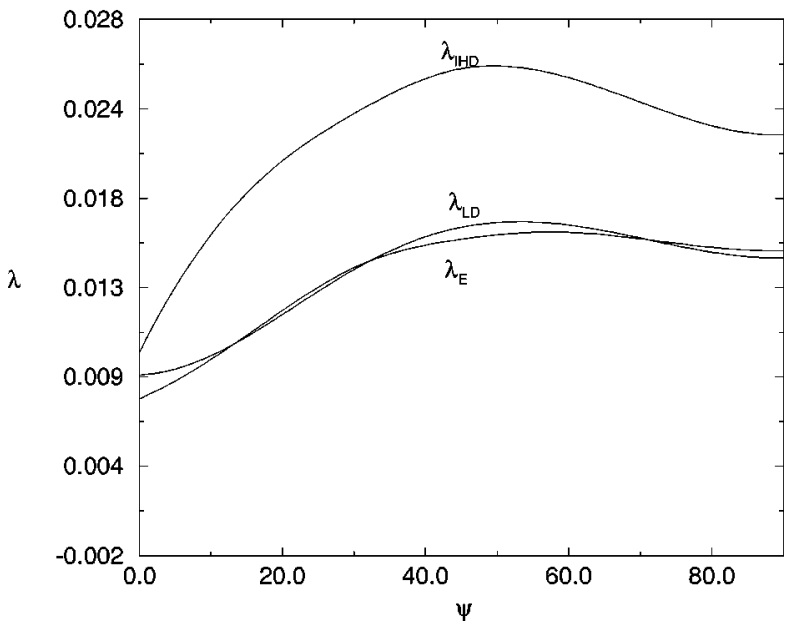

FIG. 4. Comparison of the exact value of $\lambda_{1}$, i.e., $\lambda_{E}$ with the low damping approximation of Eq. (87) $\left(\lambda_{\mathrm{LD}}\right)$, and the intermediate to high damping approximation ( $\lambda_{\mathrm{IHD}}$ ) of Eq. (68) (denoted by $\lambda_{A}$ in all other figures). The figure illustrates that in the case of low damping $(a=0.1)$ the low damping formula provides a better approximation to the exact solution.
Plot of $\lambda$ versus $\psi h=0.1, a=1.0, \sigma=10$

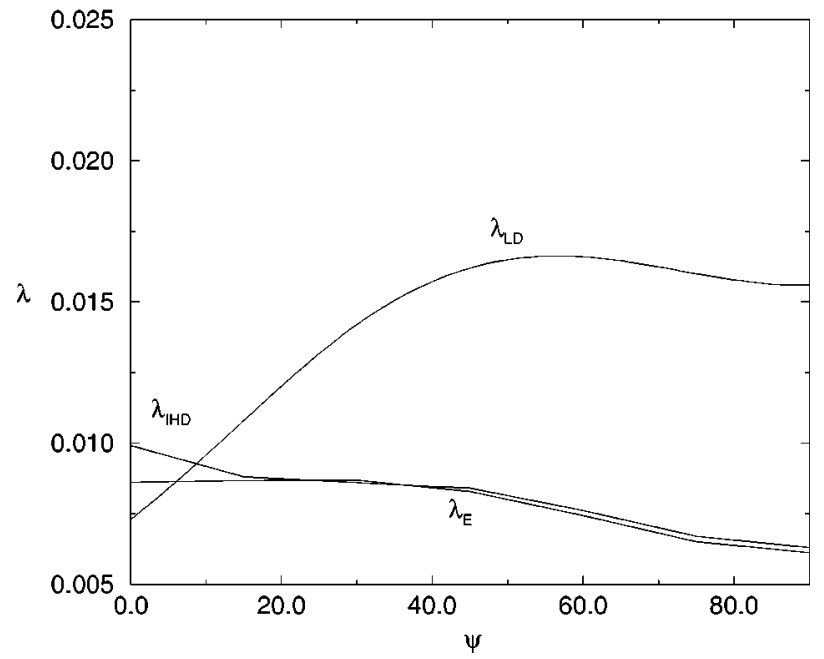

FIG. 5. Same as Fig. 4 except $a=10.0$. In this case therefore, the IHD formula of Eq. (68) provides a better approximation to the exact solution.

$\lambda_{A} / \lambda_{E}$ is in the vicinity of 1 for all $h$ values of interest. This result should be compared with one yielded by the asymptotic formula using an axially symmetric approximation as given previously by us [Eq. (82) of Ref. 12], namely,

$$
\lambda_{1}=\frac{2 \sigma(1-h) \sqrt{1+h}}{\pi h} e^{-\sigma(1-h)^{2}} .
$$

It is apparent that in the high damping limit, the two formulas differ simply by the factor $\sqrt{h}$ so that they become asymptotic to each other as $h \rightarrow 1$, the error so increasing as $h$ decreases. The effect of the $\sqrt{h}$ correction is then to eliminate the constant error apparent in Fig. 13 of Ref. 12.

However, when $a$ is very small and satisfies the criterion $a \beta\left(V_{0}-V_{2}\right) \ll 1$, then the low damping (LD) approximation formula of Eq. (87) must be used. Figure 4 illustrates the

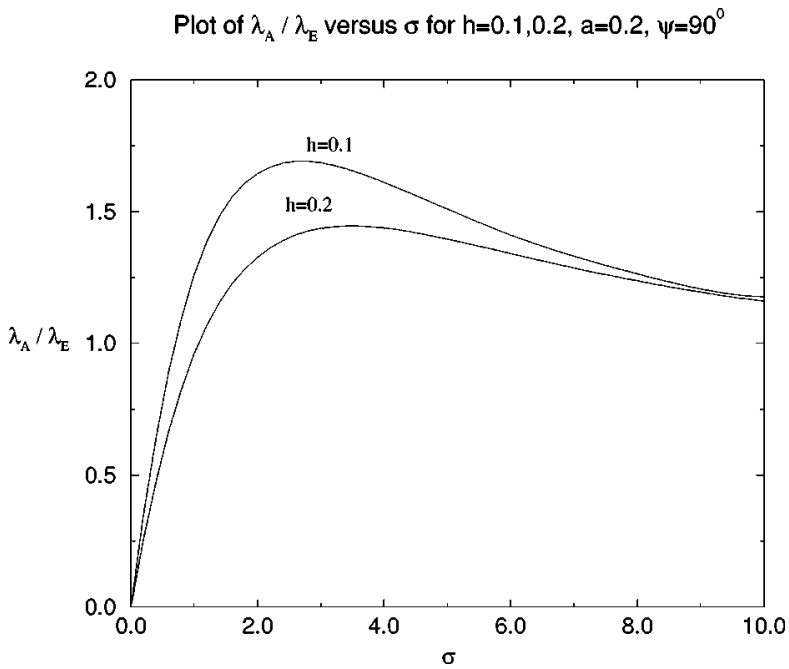

FIG. 6. Ratio of the asymptotic $\left[\lambda_{A}\right.$ yielded by Eq. (73)] and exact expressions for $\lambda_{1}$ with $a$ finite $(=0.2)$ providing reasonable constancy, and so indicating that Eq. (73) provides a useful asymptotic estimate in these cases. 
Plot of $\lambda_{\mathrm{g}} / \lambda_{\mathrm{v}}$ as a function of $\mathrm{a}, \sigma=10.0, \psi=90.0^{\circ}$

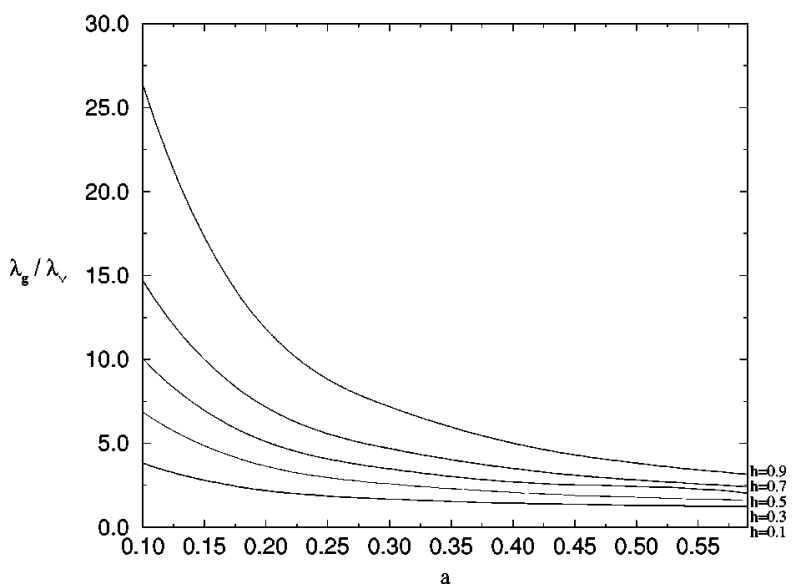

FIG. 7. Ratio of $\left[\lambda_{A}(a)=\lambda_{g}\right]$ and $\left[\lambda_{A}(\infty)=\lambda_{\nu}\right]$ showing the variation of $\lambda_{1}(a) / \lambda_{1}(\infty)$ for various values of the parameter $a$. Clearly the effect of the gyromagnetic term is to decrease the longitudinal relaxation time.

exact eigenvalue $\lambda_{E}$, the approximation given by the IHD formula $\left(\lambda_{\mathrm{IHD}}\right)$ of Eq. (68), and the approximation given by the low damping formula $\left(\lambda_{\mathrm{LD}}\right)$. On examining Fig. 4 , it is evident in this case $(a=0.1, h=0.1, \sigma=10)$, that the low damping formula provides a much better approximation to the lowest eigenvalue. Figure 5 provides a plot of $\lambda_{E}, \lambda_{\mathrm{IHD}}$, and $\lambda_{\mathrm{LD}}$ for the case when $a=1.0, h=0.1, \sigma=10$. Here the criterion $a \beta\left(V_{0}-V_{2}\right) \gg 1$ is obviously satisfied, and it is clear that in this situation, the IHD formula of Eq. (68) provides a much better approximation to the exact value of the lowest eigenvalue.

From Fig. 6 onwards, we use the IHD formula to approximate $\lambda_{1}$ (namely, $\lambda_{A}$ ). In Fig. 6, we show the ratio of the approximate value of $\lambda_{1}$ given by Eq. (68), and the exact value $\lambda_{E}\left(\lambda_{A} / \lambda_{E}\right)$ versus $\sigma$ for finite $a=0.2$. Again the consistency is reasonable indicating that Eq. (68) provides a useful asymptotic estimate in this case (i.e., as borne out by Fig. 2). In Fig. 7 we show the variation of $\lambda_{A}(a) / \lambda_{A}(\infty)$ for various values of $a$ indicating that the general effect of the gyromagnetic term is to decrease the longitudinal relaxation time.

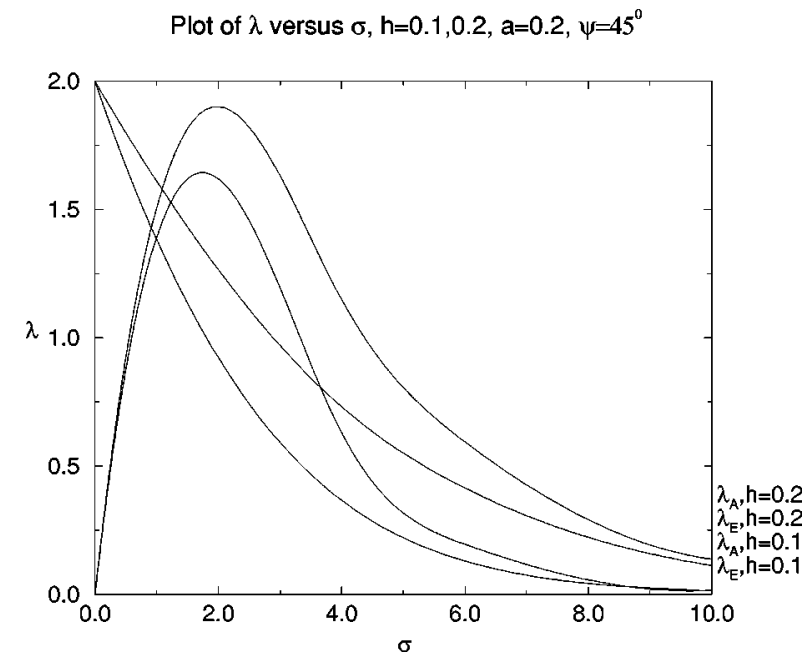

FIG. 8. Same as Fig. 2 with $\psi=45^{\circ}$ rather than $90^{\circ}$.

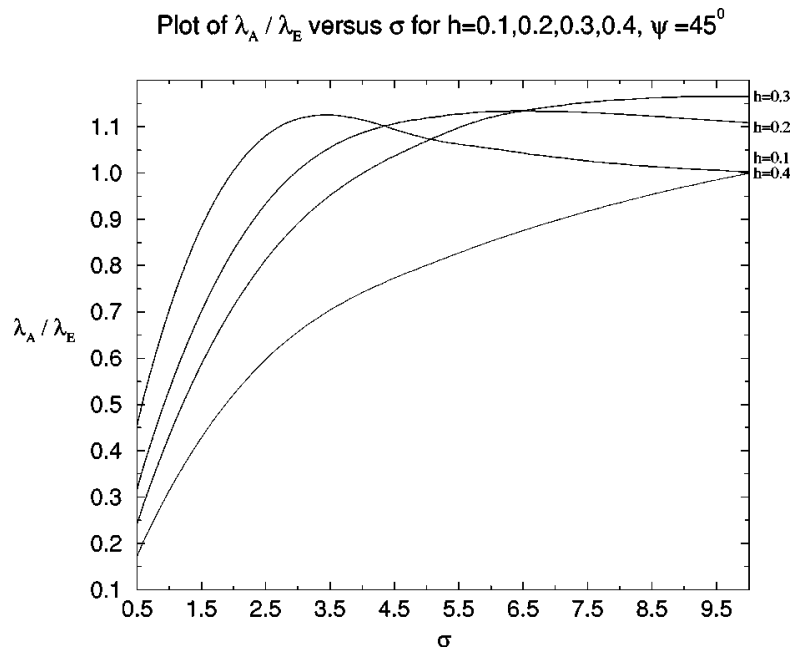

FIG. 9. Comparison of the solution $\left[\lambda_{1}(\pi / 4)\right]$ yielded by the asymptotic formula of Eq. (68) in the high damping limit $(a \rightarrow \infty)$ with the exact numerical solution, the constancy indicating that the asymptotic estimate again yields a good approximation to the exact solution.

This can be understood by means of the following discussion. Without an applied field, or with an applied field and $\psi=0$, the gyromagnetic term in the Gilbert equation [corresponding to the fourth term in Eq. (7)] has no effect on the calculation of the Néel relaxation mode, which means that the longitudinal (Néel) and the transverse (precessional) modes are completely decoupled from each other. The effect of the dimensionless damping factor $a$ on the longitudinal (Néel) relaxation mode manifests itself solely through the diffusional $\left(\tau_{N}\right)$ time, proportional to $\left(1+a^{2}\right) / a$, which leads to the disappearance of the Néel relaxation in the limiting cases ( $a=\infty$ and zero). Now, with an applied field with $\psi$ different from zero, there is a strong (mode-mode) coupling between the two modes of motion. The mode-mode coupling manifests itself as a geometric dependence of the prefactor of $\lambda_{1}$ on $a$ which for $\psi=\pi / 2$, for example, is

$$
=\frac{\sigma \sqrt{1+h}}{\pi \sqrt{h}}\left[1-2 h+\sqrt{1+4 a^{-2} h(1-h)}\right]
$$

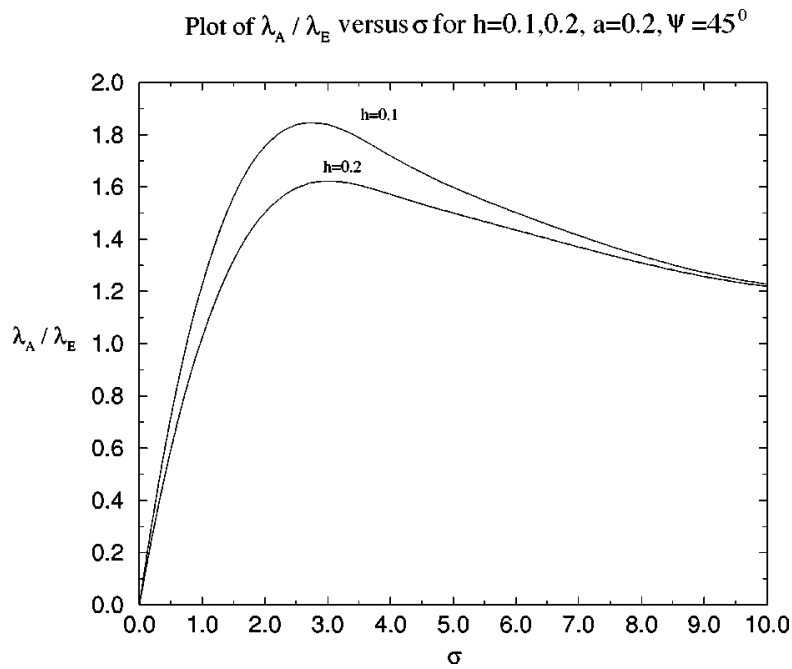

FIG. 10. Same as Fig. 6 except $\psi=45^{\circ}$. 
Plot of $\lambda_{\mathrm{g}} / \lambda_{\mathrm{v}}$ as a function of $\mathrm{a}, \psi=45^{\circ}$

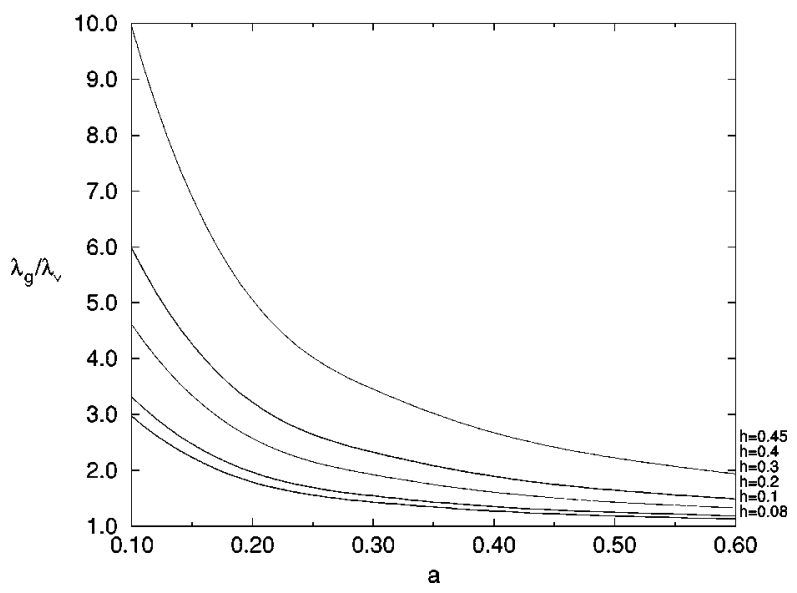

FIG. 11. Same as Fig. 7 except $\psi=45^{\circ}$.

which we shall use for the purpose of discussion. It is evident that the precessional mode corresponds to very high frequencies (that is, to very short times, much shorter than the Néel time when $\sigma$ is not too small [in fact when Eq. (73) is valid]), and that the coupling leads to a decrease of the longitudinal (Néel) time compared to the case where no coupling acts. Thus, the variation of the relaxation time of the Néel mode with $a$ is governed by two factors, namely, the diffusional time $\tau_{N}$ [proportional to $\left(1+a^{2}\right) / a$ ] and by the mode-mode coupling effect (that is the decrease of $1 / \lambda_{1}$ with a).

Figures 8-10 show the behavior of $\lambda_{E}$ for $\psi=\pi / 4$ compared with the asymptotic $\lambda_{A}$. It is apparent that the asymptotic estimate yielded by Eqs. (68), (74)-(82) again provides a good approximation to the exact solution. We remark, however, that higher values of $\sigma$ at given $h$ have to be taken in this instance in order that the asymptotic formula

Plot of $\lambda$ versus $\psi, \sigma=10.0, h=0.2, a=0.2,10^{6}$

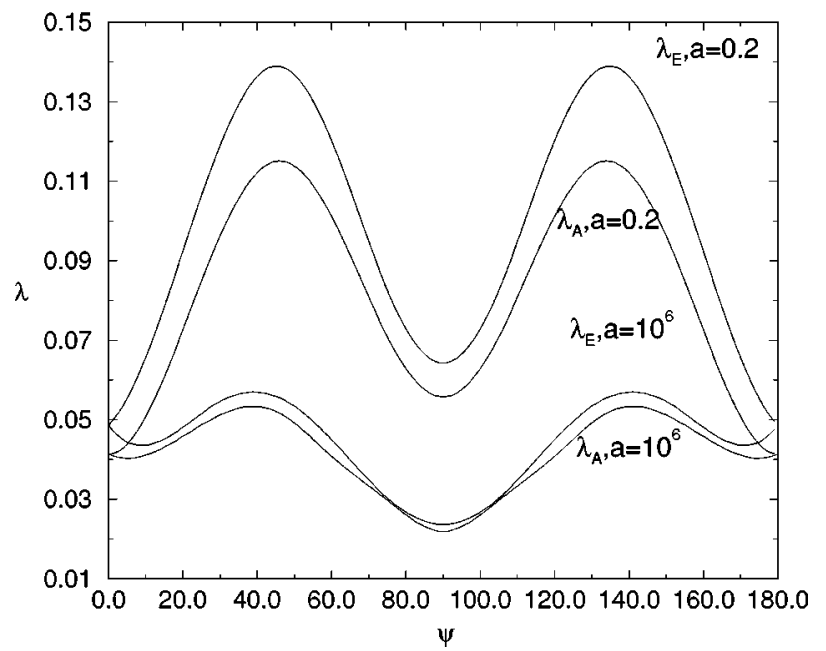

FIG. 12. Variation of the exact $\left(\lambda_{1}=\lambda_{E}\right)$ and asymptotic $\left(\lambda_{1}\right.$ $=\lambda_{A}$ ) values of $\lambda_{1}$ with $\psi$. The asymptotic estimate given by Eq. (68) provides a good approximation to the exact solution by reasonably predicting the behavior of $\lambda_{1}$. The deviation near $\psi=0^{\circ}$ is due to the fact that the asymptotic estimate of Eq. (68) is no longer valid in the region $\psi=0^{\circ}$.
Plot of $1 / \lambda$ versus $\psi, \sigma=10.0, h=0.2, a=0.2,10^{6}$

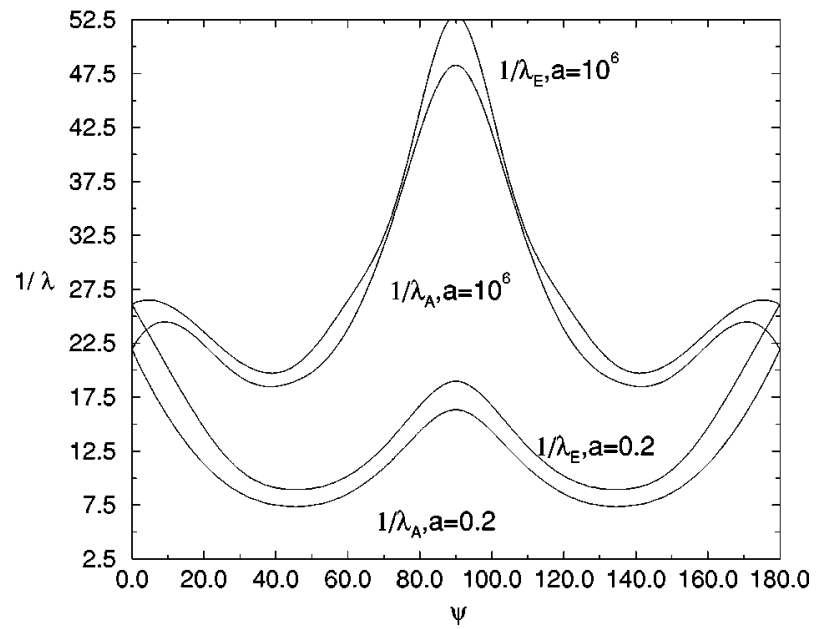

FIG. 13. Variation of the reciprocals of the exact and asymptotic values of $\lambda_{1}$ with $\psi$ showing that the Néel relaxation time is an absolute maximum at $\psi=90^{\circ}$ and a minimum at $\psi=45^{\circ}$.

should be valid as the effective barrier height is only half that for $\psi=0$ or $\psi=\pi / 2$. This makes numerical calculations more difficult to carry out as a larger matrix size must be taken in order to ensure convergence. Inspection of Fig. 11 shows that the constancy of $\lambda_{A} / \lambda_{E}$ is also remarkable for this case for values of $\sigma$ in excess of about 8 for the reduced field $h$ given. It is apparent from Figs. 9 and 10 that the systematic error induced by the axially symmetric approximation [Eqs. (101) and (102) and Fig. 14 of Ref. 12] is again eliminated by use of the correct asymptotic formula Eqs. (68), (74)-(82). In Fig. 11 we show the variation of $\lambda_{A}(a) / \lambda_{A}(\infty)$ for $\psi=\pi / 4$ with $a$.

In situations where the value of $\psi$ differs from $0, \pi / 4, \pi / 2$, the quartic Eq. (65) is solved numerically and the roots ordered using $-1 \leqslant x_{2} \leqslant x_{0} \leqslant x_{0}^{\prime} \leqslant x_{1} \leqslant 1$. The root $x_{0}^{\prime}$ corresponding to the anisotropic maximum at $\varphi=\pi$ is not re-

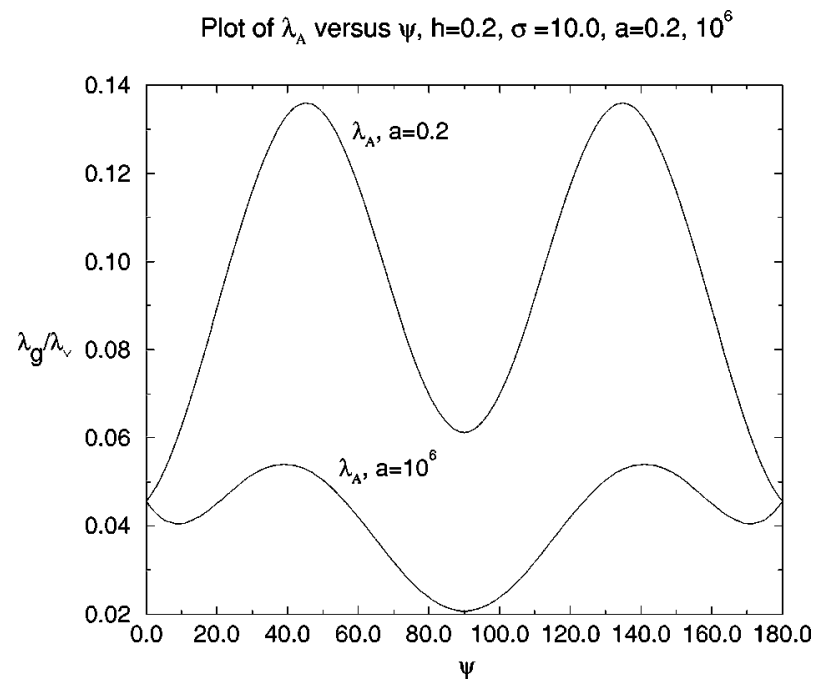

FIG. 14. Variation of the asymptotic values of $\lambda_{1}$, when $\lambda_{A}(\infty)=\lambda_{A}, a=10^{6}$, and $\lambda_{A}(0.2)=\lambda_{A}, a=0.2$ with $\psi$. The plot confirms that the effect of the gyromagnetic term is to reduce the relaxation time. 


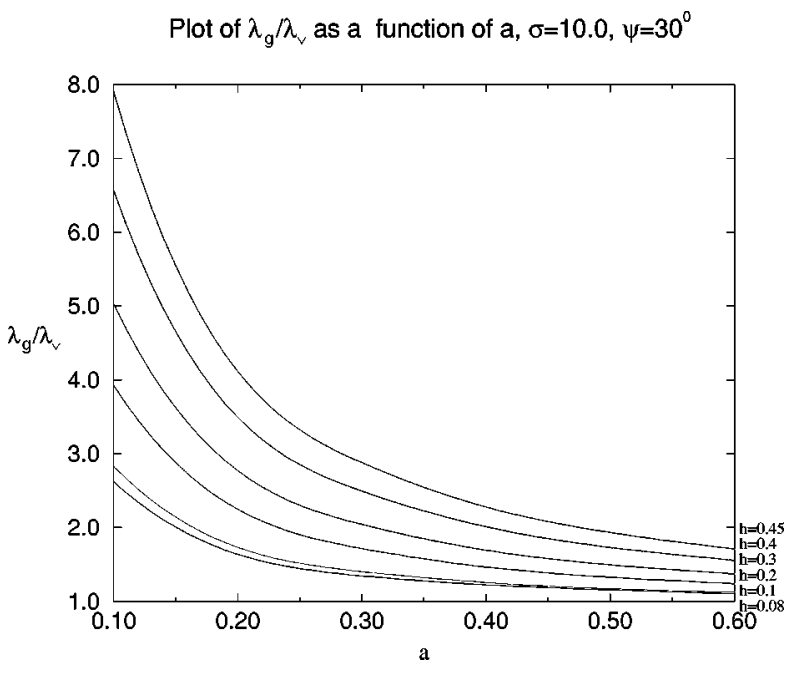

FIG. 15. Same as Figs. 7 and 11 with $\psi=30^{\circ}$.

quired. On proceeding in this way it is then possible to compare the variation of $\lambda_{1}$ with the angle $\psi$ as predicted by the exact solution with that yielded by the asymptotic estimate Eq. (68). This is shown in Fig. 12. Figure 13 which is the reciprocal of Fig. 12, shows that in all cases the Néel relaxation time has an absolute maximum at $\psi=\pi / 2$ and a minimum near $\psi=\pi / 4$. It is apparent that the asymptotic formula Eq. (68) again provides a reasonable estimate of $\lambda_{1}$. The deviation near $\psi=0$ is consistent with the fact that the asymptotic estimate, Eq. (68), is no longer valid in the region $\psi=0$. The asymptote should be constructed in this case using the Brown-Aharoni formula Eq. (69). This formula does not exhibit the characteristic $\sqrt{h}$ dependence of the nonaxially symmetric asymptote neither does it contain the dimensionless damping factor $a$ arising from the inclusion of the gyromagnetic term which occurs when one uses Eq. (68) so invalidating that formula for very small values of $h \ll 0.1$, and for $\psi=0$ also invalidating Eq. (68) as there is no $a$ dependence in this axially symmetric situation. In view of the acceptable approximation provided by the asymptotic formula of Eq. (68), we have shown in Fig. 14 the variation of $\lambda_{A}$ with $\psi$ for $a=\infty$ and $a=0.2$ confirming that the effect

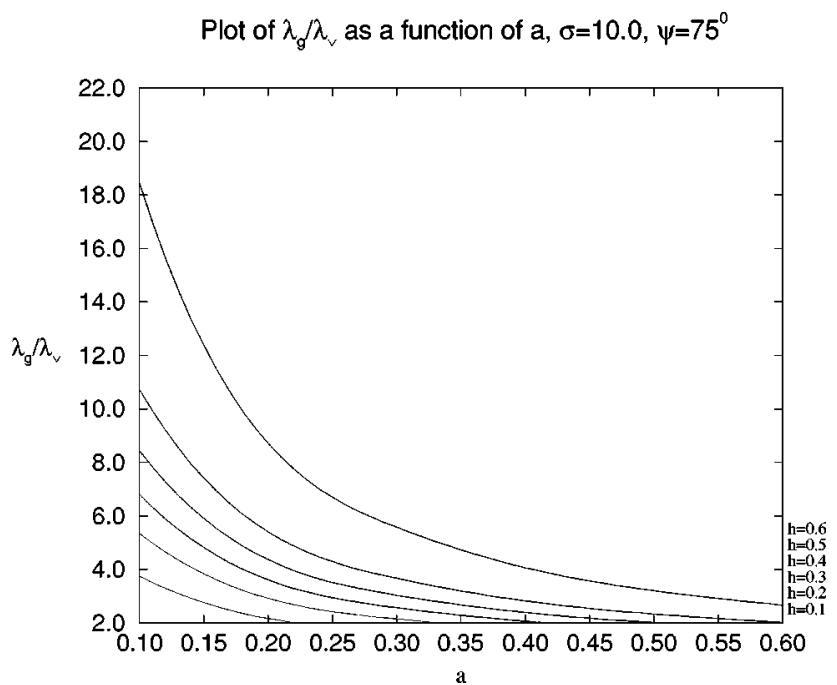

FIG. 16. Same as Figs. 7, 11, and 15 with $\psi=75^{\circ}$.
TABLE I. The lowest nonvanishing eigenvalue $\lambda_{1}$ for various values of the barrier height $(\sigma)$ and field parameters $(h) ; \psi=0^{\circ}$.

\begin{tabular}{lcccccc}
\hline \hline & & $\lambda_{1}$ & & \\
$h$ & $\sigma=0.2$ & $\sigma=0.5$ & $\sigma=1.0$ & $\sigma=2.0$ & $\sigma=5.0$ & $\sigma=10.0$ \\
\hline 0.01 & 1.844 & 1.627 & 1.306 & 0.8079 & 0.1354 & 0.002920 \\
0.1 & 1.845 & 1.629 & 1.314 & 0.8322 & 0.1876 & 0.00860 \\
0.2 & 1.846 & 1.634 & 1.336 & 0.9056 & 0.3134 & 0.03830 \\
0.4 & 1.849 & 1.659 & 1.426 & 1.196 & 0.9109 & 0.3846 \\
0.5 & 1.852 & 1.676 & 1.493 & 1.412 & 1.398 & 0.8829 \\
0.7 & 1.860 & 1.723 & 1.671 & 1.974 & 2.782 & 2.927 \\
0.8 & 1.864 & 1.753 & 1.783 & 2.317 & 3.670 & 4.524 \\
1.0 & 1.876 & 1.823 & 2.049 & 3.121 & 5.824 & 8.777 \\
\hline \hline
\end{tabular}

of the gyromagnetic term is to reduce the relaxation time. Finally in Figs. 15 and 16 we show the variation of $\lambda_{A}(a) / \lambda_{A}(\infty)$ with $a$ as computed for $\psi=30^{\circ}$ and $\psi=75^{\circ}$ using the asymptotic formula Eq. (68).

The results we have just given confirm that the asymptotic formula following the Brown ${ }^{10}$ approach [Eq. (68)] is capable of providing an accurate description of the Néel relaxation time for the present nonaxially symmetric problem in the IHD limit [where the criterion $a \beta\left(V_{0}-V_{2}\right) \gg 1$ is satisfied]. Brown's approach which appears to have been suggested by the earlier work of Smith and de Rozario ${ }^{19}$ is in effect $^{3}$ an adaptation to spin relaxation of the intermediate to high damping asymptotic formula of Kramers ${ }^{14}$ [also Eq. (25) of Ref. 14] for escape rates for a process governed by the Fokker-Planck equation in phase space (the KleinKramers equation ${ }^{14}$ ) to the present problem. In the limit of very small damping Eq. (68) will reduce to a formula analogous to Eq. (26) of Kramers ${ }^{14}$ (the transition state value in his nomenclature). This is unphysical, since it indicates that Néel relaxation can take place in the limit of zero friction and it is an artifact of applying the IHD formula beyond its range of validity. In order to discuss the behavior at small $a$, the low friction Kramers formula must be used. In general, the IHD theory may only be applied when the energy loss during one period of the undamped motion (i.e., precession) is $>k T$. If this criterion is not satisfied, the moderately damped formula must be used [controlled by $a \beta\left(V_{0}-V_{2}\right)$ ].

A discussion of the problem in the context of the Kramers ${ }^{14}$ calculation has also been given by Klik and Gunther. $^{22}$ In order to further assist the reader we show in the

TABLE II. The lowest nonvanishing eigenvalue $\lambda_{1}$ for various values of the barrier height $(\sigma)$ and field parameters $(h) ; \psi=30^{\circ}$.

\begin{tabular}{lcccccc}
\hline \hline & & $\lambda_{1}$ & & \\
$h$ & $\sigma=0.2$ & $\sigma=0.5$ & $\sigma=1.0$ & $\sigma=2.0$ & $\sigma=5.0$ & $\sigma=10.0$ \\
\hline 0.01 & 1.844 & 1.627 & 1.306 & 0.8079 & 0.1358 & 0.002924 \\
0.1 & 1.845 & 1.629 & 1.313 & 0.8297 & 0.1743 & 0.008450 \\
0.2 & 1.845 & 1.634 & 1.334 & 0.8691 & 0.306 & 0.04819 \\
0.4 & 1.850 & 1.656 & 1.416 & 1.165 & 1.022 & 0.8447 \\
0.5 & 1.852 & 1.672 & 1.477 & 1.371 & 1.706 & 2.248 \\
0.7 & 1.859 & 1.716 & 1.641 & 1.929 & 3.905 & 8.499 \\
0.8 & 1.863 & 1.743 & 1.744 & 2.284 & 5.447 & 13.50 \\
1.0 & 1.874 & 1.808 & 1.989 & 3.148 & 9.349 & 26.94 \\
\hline \hline
\end{tabular}


TABLE III. The lowest nonvanishing eigenvalue $\lambda_{1}$ for various values of the barrier height $(\sigma)$ and field parameters $(h) ; \psi=45^{\circ}$.

\begin{tabular}{lcccccc}
\hline \hline \multicolumn{7}{c}{$\lambda_{1}$} \\
& $\sigma=0.2$ & $\sigma=0.5$ & $\sigma=1.0$ & $\sigma=2.0$ & $\sigma=5.0$ & $\sigma=10.0$ \\
\hline 0.01 & 1.844 & 1.627 & 1.306 & 0.8079 & 0.1357 & 0.002918 \\
0.1 & 1.845 & 1.628 & 1.312 & 0.8272 & 0.1694 & 0.007901 \\
0.2 & 1.845 & 1.634 & 1.331 & 0.8861 & 0.2895 & 0.04788 \\
0.4 & 1.849 & 1.654 & 1.405 & 1.127 & 0.9981 & 0.9523 \\
0.5 & 1.851 & 1.669 & 1.461 & 1.313 & 1.712 & 2.594 \\
0.7 & 1.858 & 1.708 & 1.609 & 1.825 & 4.108 & 10.05 \\
0.8 & 1.862 & 1.733 & 1.701 & 2.153 & 5.850 & 16.22 \\
\hline \hline
\end{tabular}

manner of Tables I-IV, a representative selection of $\lambda_{1}$ for various angles and for $a=\infty$. We shall now proceed to the calculation of the correlation time.

\section{NUMERICAL CALCULATION OF THE CORRELATION TIME}

The results of the numerical calculation of the correlation time are shown in Figs. 17-20 and in Tables V-VIII. First, the equilibrium values are obtained by solving Eq. (42), then the initial values are obtained by means of Eq. (46), and finally the zero frequency Laplace transforms are obtained by solving Eq. (48). The correlation time is then given by Eq. (55). An alternative procedure is to construct the matrix in Eq. (50) from the eigenvectors of $A$ and solve Eq. (52). On calculating the amplitudes in Eq. (54), the correlation time is then given by Eq. (18).

It is then apparent from Figs. 17-20 that the correlation time always exhibits significant departure from increasing exponential behavior for large $\sigma$ where $h$ is sufficiently large, however, far less than the critical value needed for the destruction of the bistable nature of the potential. This conclusion is reinforced by the results shown in Figs. 21 and 22 where in all cases the product of the correlation time and the smallest eigenvalue shows a very marked departure from unity for sufficiently large $h$ and $\sigma$. Moreover, the effect has a strong angle dependence, the most marked departure being at $\psi=\pi / 2$. This is a striking example of the effect of a strong uniform field which was first described in Ref. 33, and explained physically by Garanin ${ }^{34}$ as being due to the depletion of the shallower of the two potential wells by the field so that

TABLE IV. The lowest nonvanishing eigenvalue $\lambda_{1}$ for various values of the barrier height $(\sigma)$ and field parameters $(h) ; \psi=90^{\circ}$.

\begin{tabular}{lcccccc}
\hline \hline & & $\lambda_{1}$ & & \\
$h$ & $\sigma=0.2$ & $\sigma=0.5$ & $\sigma=1.0$ & $\sigma=2.0$ & $\sigma=5.0$ & $\sigma=10.0$ \\
\hline 0.01 & 1.844 & 1.627 & 1.306 & 0.8073 & 0.1356 & 0.002906 \\
0.1 & 1.845 & 1.628 & 1.311 & 0.8220 & 0.1577 & 0.005649 \\
0.2 & 1.845 & 1.632 & 1.326 & 0.8649 & 0.2276 & 0.01889 \\
0.4 & 1.848 & 1.649 & 1.383 & 1.034 & 0.5392 & 0.1676 \\
0.5 & 1.850 & 1.661 & 1.426 & 1.158 & 0.7968 & 0.3918 \\
0.7 & 1.856 & 1.693 & 1.540 & 1.478 & 1.5354 & 1.384 \\
0.8 & 1.859 & 1.714 & 1.609 & 1.671 & 2.014 & 2.198 \\
1.0 & 1.867 & 1.762 & 1.774 & 2.114 & 3.167 & 4.585 \\
\hline \hline
\end{tabular}

Plot of the Reciprocal of the Correlation time versus $\sigma, \psi=0^{\circ}$

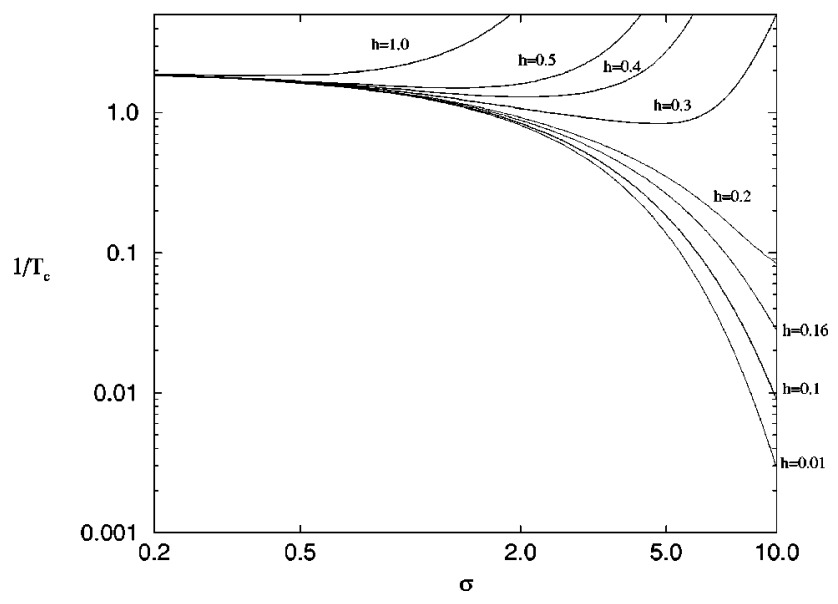

FIG. 17. Variation of the reciprocal of the correlation time with the barrier height parameter $\sigma$ for $\psi=0^{\circ}$. The correlation time exhibits significant departure from increasing exponential behavior as $\sigma$ increases when $h$ is large (but no larger than $h_{c}$ at which point the bistable structure of the potential is destroyed).

the Néel mode is completely swamped by the relatively fast modes in the deeper potential well.

The behavior of the correlation time $T_{c}$ for small values of $h$ is similar to that of $1 / \lambda_{1}$, except for values of $\psi$ in the range of $60^{\circ}-90^{\circ}$, where a strong decrease is observed with $T_{c}$ becoming very small. This is also true of the product $T_{c} \lambda_{1}$ (Figs. 21,22) and may be explained as follows. For $\psi$ $=\pi / 2$, the two minima of the energy are symmetric with respect to the direction of the applied field and so their populations are equal in thermodynamic equilibrium. Starting from such a situation, on slightly decreasing the field, there is a quasi-instantaneous small change of the angle of the minima and thus the magnetic moment direction (so that only rapidly damped fast oscillations occur). Thus, the area under the magnetization decay curve, i.e., $T_{c}$ is very small. In fact $\chi_{\|} \approx 0$ while $\chi_{\perp}$ maintains its oscillatory character. On the other hand when $\psi$ decreases from $90^{\circ}$, the two minima become asymmetric with respect to the applied field direction, and the decay of the magnetization appears along

Plot of the Reciprocal of the Correlation time versus $\sigma, \psi=45^{\circ}$

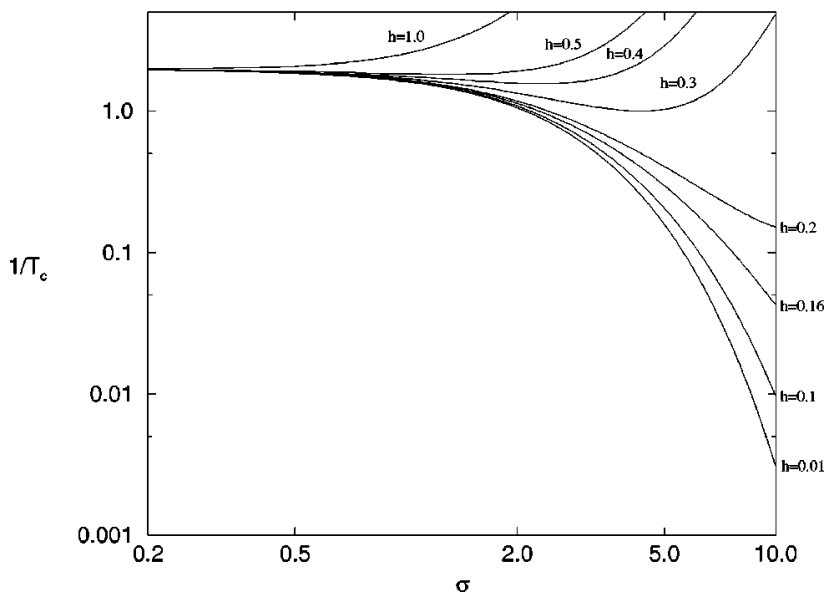

FIG. 18. Same as Fig. 17 with $\psi=45^{\circ}$. 
Plot of the Reciprocal of the Correlation Time versus $\sigma, \psi=60^{\circ}$

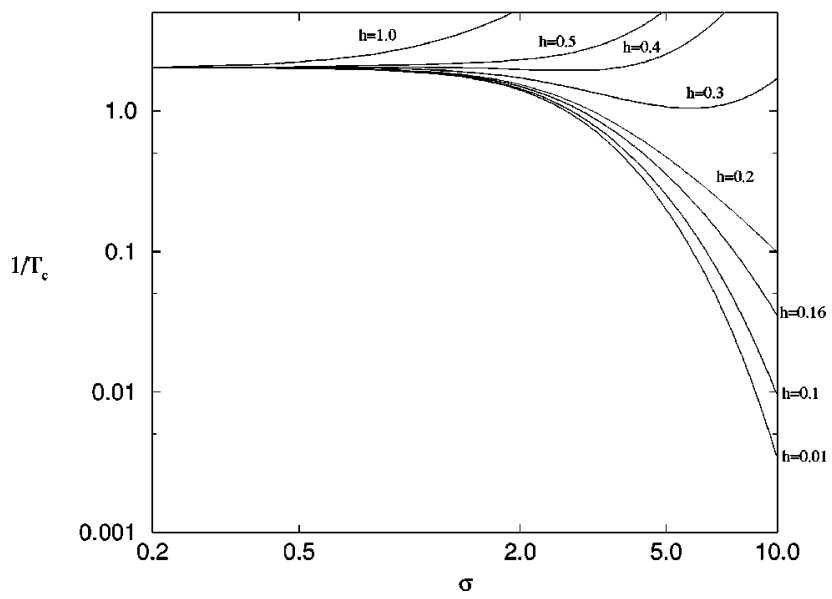

FIG. 19. Same as Fig. 17 with $\psi=60^{\circ}$.

this direction. Here $T_{c}$ increases, and takes on values of the order of $1 / \lambda_{1}$ at $\psi \approx 75^{\circ}$.

In fact, for $\psi \leqslant 70^{\circ}$, the product $T_{c} \lambda_{1}$ has only a weak dependence on the value of the dimensionless damping factor $a$, a slight increase being observed for small values of $\sigma$. As far as the dependence on the other parameters is concerned, $T_{c} \lambda_{1}$ decreases from unity when $\psi$ increases, with an enhanced decrease if $\sigma$ is small. On the other hand, $T_{c} \lambda_{1}$ slightly increases with $\sigma$, and finally $T_{c} \lambda_{1}$ is almost independent of $h$ for small values of $\sigma$, while it decreases for high values of $\sigma$.

We wish to emphasize that values of $T_{c} \lambda_{1}$ sensibly smaller than unity indicate that the higher (faster) relaxation modes (corresponding to $\lambda_{2}, \lambda_{3}, \ldots$ ) are of importance. Nevertheless for those experiments which measure the decay of the magnetization over a long period of time, such as field cooled and zero field cooled magnetizations, and thermoremanent magnetization experiments, $\lambda_{1}$ is the correct parameter because the magnetization is governed by

Plot of the Reciprocal of the Correlation time versus $\sigma, \psi=90^{\circ}$

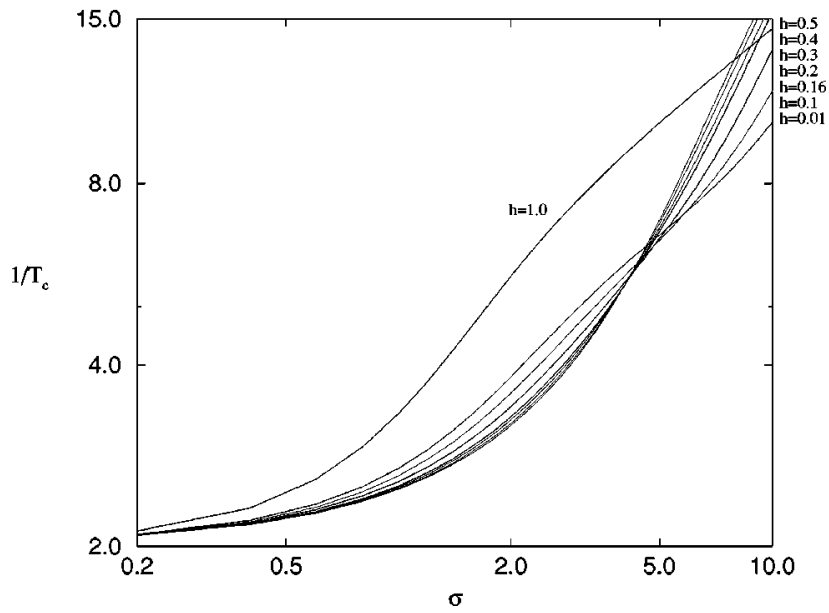

FIG. 20. Variation of the reciprocal of the correlation time with the barrier height parameter $\sigma$ for the case when $\psi=90^{\circ}$. The correlation time exhibits the most significant departure from exponential behavior at $\psi=90^{\circ}$.
TABLE V. Reciprocal of the correlation time $1 / T_{c}$ for various values of the barrier height $(\sigma)$ and the field $(h)$ parameters; $\psi$ $=0^{\circ}$.

\begin{tabular}{lcccc}
\hline \hline & \multicolumn{5}{c}{$1 / T_{c}$} \\
$h$ & $\sigma=1.0$ & $\sigma=2.0$ & $\sigma=5.0$ & $\sigma=10.0$ \\
\hline 0.01 & 1.309 & 0.8132 & 0.1375 & 0.002943 \\
0.1 & 1.317 & 0.8404 & 0.1833 & 0.008923 \\
0.2 & 1.343 & 0.9246 & 0.3496 & 0.08420 \\
0.4 & 1.446 & 1.292 & 2.738 & 43.33 \\
0.5 & 1.526 & 1.607 & 9.160 & 51.79 \\
0.7 & 1.744 & 2.648 & 24.58 & 60.75 \\
0.8 & 1.886 & 3.451 & 28.05 & 65.11 \\
1.0 & 2.244 & 5.728 & 33.17 & 73.68 \\
\hline \hline
\end{tabular}

$$
\sum_{k} A_{k} e^{-\lambda_{k} t / 2 \tau_{N}}
$$

and as $\lambda_{1}<\lambda_{k}(k \geqslant 2)$, the term in $e^{-\lambda_{1} t / 2 \tau_{N}}$ (that is, the longest lived relaxation mode) dominates. However, in those experiments which measure the magnetization behavior over a short time interval (such as susceptibility measurements at high frequency), $T_{c}$ must be used. Consequently if $T_{c} \lambda_{1}$ is markedly different from unity, then measurements at short and long times do not examine the same phenomena so that the results are not directly comparable.

As far as the numerical calculations are concerned, the exact values of the correlation time were computed by setting up the $A$ matrix of coefficients as in the calculation of $\lambda_{1}$, and also the vector $B$, as given by Eq. (39). A LAPACK linear equation solver was then used to compute the solution to the real system of linear equations

$$
A U_{\infty}=-B \text {, }
$$

as given by Eq. (44), where $L U$ decomposition with partial pivoting and row interchanges is used to factor $A$ as

$$
A=P L U,
$$

where $P$ is a permutation matrix, $L$ is a unit lower triangular, and $U$ is an upper triangular. The factored form of $A$ was then used to solve the system of equations $A U_{\infty}=-B$. The next step was to set up the matrix $W$ as described in Sec. IV,

TABLE VI. Reciprocal of the correlation time $1 / T_{c}$ for various values of the barrier height $(\sigma)$ and the field $(h)$ parameters; $\psi$ $=30^{\circ}$.

\begin{tabular}{lcccc}
\hline \hline & \multicolumn{5}{c}{$1 / T_{c}$} \\
$h$ & $\sigma=1.0$ & $\sigma=2.0$ & $\sigma=5.0$ & $\sigma=10.0$ \\
\hline 0.01 & 1.432 & 0.8989 & 0.1444 & 0.002998 \\
0.1 & 1.440 & 0.9269 & 0.1906 & 0.009579 \\
0.2 & 1.466 & 1.014 & 0.3785 & 0.1647 \\
0.4 & 1.571 & 1.396 & 3.102 & 27.49 \\
0.5 & 1.652 & 1.720 & 8.464 & 30.96 \\
0.7 & 1.872 & 2.756 & 18.31 & 35.75 \\
0.8 & 2.015 & 3.518 & 20.54 & 38.16 \\
1.0 & 2.371 & 5.560 & 23.88 & 43.09 \\
\hline \hline
\end{tabular}


TABLE VII. Reciprocal of the correlation time $1 / T_{c}$ for various values of the barrier height $(\sigma)$ and the field $(h)$ parameters; $\psi$ $=45^{\circ}$.

\begin{tabular}{lcccc}
\hline \hline & \multicolumn{5}{c}{$1 / T_{c}$} \\
$h$ & $\sigma=1.0$ & $\sigma=2.0$ & $\sigma=5.0$ & $\sigma=10.0$ \\
\hline 0.01 & 1.614 & 1.053 & 0.1582 & 0.003109 \\
0.1 & 1.623 & 1.083 & 0.2053 & 0.009641 \\
0.2 & 1.649 & 1.175 & 0.4052 & 0.1510 \\
0.4 & 1.756 & 1.576 & 2.892 & 22.26 \\
0.5 & 1.837 & 1.909 & 6.923 & 26.33 \\
0.7 & 2.059 & 2.919 & 14.77 & 29.85 \\
0.8 & 2.201 & 3.621 & 16.75 & 36.62 \\
\hline \hline
\end{tabular}

and then the initial values vector $C_{0}$ was evaluated as in Eq. (47). The zero frequency Laplace transform vector $\widetilde{C}(0)$ is then computed by again using the LAPACK linear equation solver DGESV, and the correlation time was found by using the zero frequency Laplace transform vector as indicated by Eq. (55). The matrix size parameter lmax was increased until successive results were obtained within a tolerance of at least $10^{-4}$. All programs were again coded in FORTRAN, and all results were obtained using the CRAY-J90 at the Rutherford Appleton Laboratory, requiring no more than two minutes of CPU time for even the most difficult cases. For example, when $\psi=\pi / 4, h=0.4, a=0.2$, and $\sigma=10$, the amount of CPU time required to complete the calculation for successive matrix sizes given by $\operatorname{lmax}=25$ and $\operatorname{lmax}=26$, within a tolerance of $10^{-7}$ was $78.01 \mathrm{sec}$.

\section{GENERAL DISCUSSION AND CONCLUSIONS}

In this paper we have given methods for the calculation of the Néel relaxation time and the integral relaxation time (here, the correlation time) for an assembly of noninteracting superparamagnetic particles with the field applied at an oblique angle to the easy axis of magnetization (and including the gyromagnetic term) by direct solution of the simultaneous linear equations which arise from the Fokker-Planck equation.

In general terms, the behavior shows a departure of the Néel relaxation time from the integral relaxation time similar to that of Coffey, Crothers, Kalmykov, and Waldron, ${ }^{33}$ for an

TABLE VIII. Reciprocal of the orrelation time $1 / T_{c}$ for various values of the barrier height $(\sigma)$ and the field $(h)$ parameters; $\psi$ $=90^{\circ}$.

\begin{tabular}{lcccccc}
\hline \hline \multicolumn{7}{c}{$1 / T_{c}$} \\
$h$ & $\sigma=0.2$ & $\sigma=0.5$ & $\sigma=1.0$ & $\sigma=2.0$ & $\sigma=5.0$ & $\sigma=10.0$ \\
\hline 0.01 & 2.083 & 2.220 & 2.484 & 3.172 & 6.949 & 17.31 \\
0.1 & 2.084 & 2.222 & 2.492 & 3.198 & 6.860 & 16.81 \\
0.2 & 2.084 & 2.229 & 2.517 & 3.277 & 6.657 & 15.37 \\
0.4 & 2.089 & 2.255 & 2.617 & 3.588 & 6.434 & 11.38 \\
0.5 & 2.092 & 2.274 & 2.692 & 3.818 & 6.575 & 10.10 \\
0.7 & 2.100 & 2.327 & 2.892 & 4.415 & 7.448 & 9.975 \\
0.8 & 2.106 & 2.340 & 3.018 & 4.776 & 8.162 & 10.938 \\
1.0 & 2.118 & 2.439 & 3.318 & 5.608 & 10.08 & 14.441 \\
\hline \hline
\end{tabular}

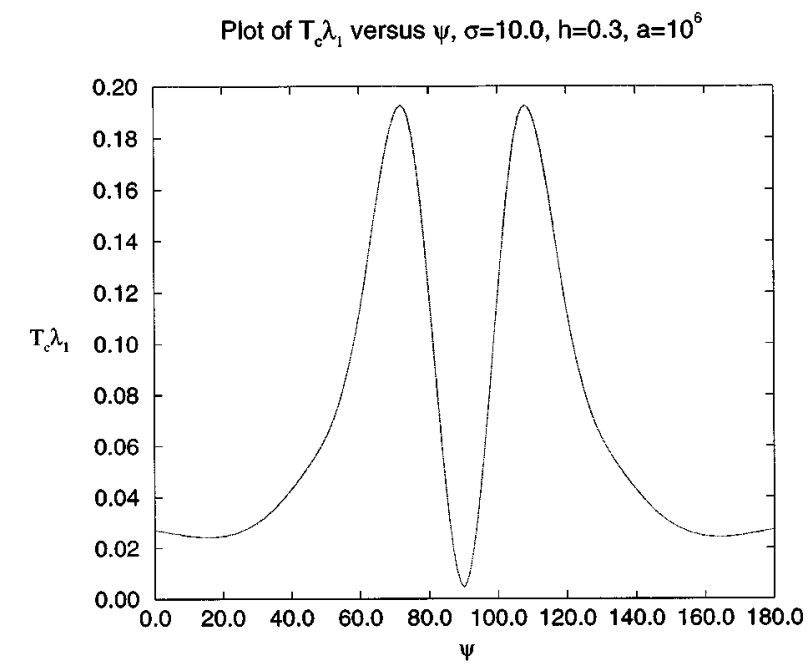

FIG. 21. Variation of the product of the exact values of the lowest eigenvalue $\lambda_{1}$ and the correlation time $T_{c}$ with $\psi$. The figure shows significant departure from the increasing exponential behavior at $\psi=90^{\circ}$.

applied field parallel to the easy axis. In addition it has been demonstrated that the asymptotic expressions for the Néel time derived by Brown ${ }^{10,15}$ in the IHD limit, and by Klik and Gunther ${ }^{22}$ in the LD limit provide a good description of the exact eigenvalue for high barriers. Both formulas are good approximations, even in the region of $a \beta\left(V_{0}-V_{2}\right) \approx 1$. A disadvantage of the present method is that it is expensive in terms of CPU time because of the large matrices which are required. Reformulation of the solution of the set of differential recurrence relations as a matrix continued fraction suggests that the size of the matrices involved may be significantly reduced, thus enabling one to carry out the calculation for much larger values of the anisotropy parameter. ${ }^{35}$ Finally we wish to emphasize the importance of the approximation formula in Eq. (68). It yields a close approximation to the exact solution (Figs. 2, 3, 6, 8-10, 12, and 13) with the exception of small values of $\sigma(\sigma \leqslant 3)$ and very small $h$ values $(h \leqslant 0.05)$. From an experimental point of view, the

Plot of $T_{c} \lambda_{1}$ versus $\psi, h=0.2, a=0.2,0.3,0.4,1.0, \sigma=10.0$

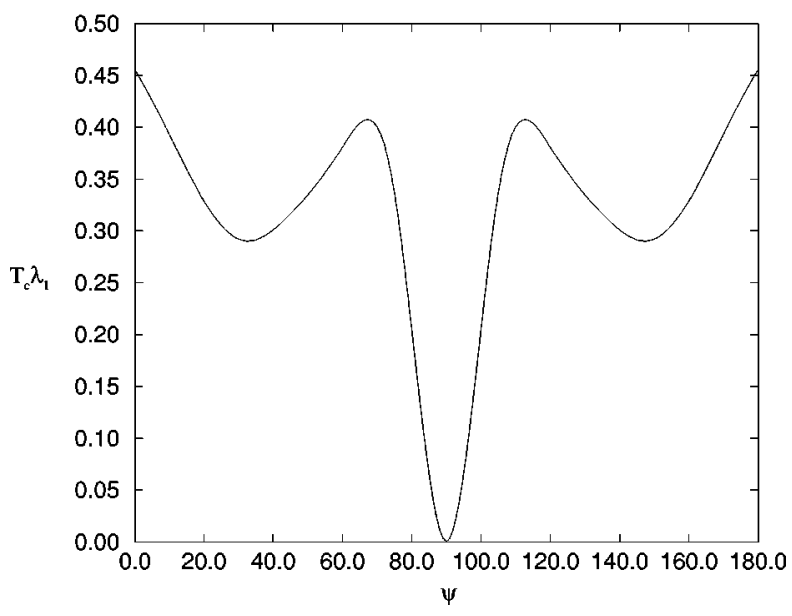

FIG. 22. Variation of $T_{c} \lambda_{1}$ with $\psi$ when the gyromagnetic term is included for various values of $a$. The figure shows that the gyromagnetic term has no effect on the behavior of $T_{c} \lambda_{1}$. 
former situation $(\sigma \leqslant 3)$ corresponds to very short relaxation times which are very difficult to observe (except by neutron dispersion), while the latter $(h \leqslant 0.05)$ occurs in examples such as field cooled and two field cooled magnetization experiments at small applied fields. Secondly, for applications of magnetic measurements, two quantities are required, not only the Néel time $\tau=2 \tau_{N} / \lambda_{1}$, but also its constituent parts $\tau^{+}, \tau^{-}$corresponding to the jump from the lower minimum to the upper minimum and the reverse jump, respectively. The reversed magnetization results from a combination of these two quantities taking account of the particular measuring process. ${ }^{1}$ For example, the field cooled magnetization $M_{\mathrm{FC}}$ is equal in the two level framework to

$$
M_{\mathrm{FC}}=M_{S R} F(T), \quad T>T_{B}=M_{S R} F\left(T_{B}\right), \quad T<T_{B}
$$

with

$$
F(T)=\frac{\tau^{+} \cos \left(\theta_{1}-\psi\right)+\tau^{-} \cos \left(\theta_{2}-\psi\right)}{\tau^{+}+\tau^{-}}
$$

and

$$
\cos \theta_{1}=x_{1}, \quad \cos \theta_{2}=x_{2} .
$$

where $T_{B}$ is the blocking temperature relative to the cooling rate, calculated from the expression for $\tau^{1}$.

It is easy to recognize in Eq. (68), the quantities $\lambda_{1}^{+}$ $\left(=2 \tau_{N} / \tau^{+}\right)$and $\lambda_{1}^{-}\left(=2 \tau_{N} / \tau^{-}\right)$which are the terms in $e^{-\beta\left(V_{0}-V_{1}\right)}$ and $e^{-\beta\left(V_{0}-V_{2}\right)}$. We remark that in the $F(T)$ calculation, the prefactor

$$
\frac{-\left(c_{1}^{(0)}+c_{2}^{(0)}\right)+\sqrt{\left(c_{2}^{(0)}-c_{1}^{(0)}\right)^{2}-4 a^{-2} c_{1}^{(0)} c_{2}^{(0)}}}{4 \pi \sqrt{-c_{1}^{(0)} c_{2}^{(0)}}}
$$

disappears, which is not so in the $\tau$ calculation. This means that for $T>T_{B}$, the $M_{\mathrm{FC}}$ values are independent of $a$ which indicates that the superparamagnetic state has been attained.

Therefore by solving the quartic Eq. (65) for a given $\psi$, we can calculate $x_{1}, x_{2}, x_{0}$, and all the $c$ 's so that we can determine $\tau, T_{B}$, and $F(T)$. We remark that in an actual sample, the field angle $\psi$ and the particle volume $v$ are random so that averages will be required, which are not too difficult to evaluate numerically on account of the simplicity of the above formulas. The above analysis may be applied directly in experimental situations such as those discussed in Refs. 7, 8, and 36 where single particles have been isolated with the intention of studying macroscopic quantum tunneling.

\section{ACKNOWLEDGMENTS}

W.T.C. acknowledges the Forbairt Research Collaboration Fund, the French Ministry for Foreign Affairs, and J.L.D., the Forbairt/C.N.R.S. Scheme for financial support for his work. D.S.F.C. acknowledges the United Kingdom EPSRC rolling grant numbers GR/L06225 and GR/L21891, L.J.G. acknowledges the E.C. Human Capital and Mobility Fund, and E.C.K. acknowledges financial support from QUB, NIDevR/ESF.
APPENDIX A: MATRIX ELEMENTS FOR THE SPHERICAL HARMONIC REPRESENTATION

$$
\begin{gathered}
e_{l, m, l, m}=-l(l+1)+\frac{2 \sigma}{(2 l-1)(2 l+3)}\left[l(l+1)-3 m^{2}\right] \\
-2 \text { uuia }^{-1} m, \\
e_{l, m, l-1, m-1}=\frac{\sigma \nu(l+1)(l+m)(l+m-1)}{(2 l+1)} \\
e_{l, m, l, m-1}=\frac{i \sigma \nu(l+m)(l-m+1)}{a}
\end{gathered}
$$

$$
e_{l, m, l+1, m-1}=\frac{\sigma \nu l(l-m+2)(l-m+1)}{(2 l+1)},
$$

$$
e_{l, m, l-2, m}=\frac{2 \sigma(l+1)(l+m)(l+m-1)}{(2 l-1)(2 l+1)},
$$

$$
e_{l, m, l-1, m}=\frac{2 \sigma(l+m)}{(2 l+1)}\left[u(l+1)-i a^{-1} m\right]
$$$$
e_{l, m, l+1, m}=-\frac{2 \sigma(l-m+1)}{(2 l+1)}\left[u l+i a^{-1} m\right],
$$$$
e_{l, m, l+2, m}=-\frac{2 \sigma(l-m+2)(l-m+1)}{(2 l+1)(2 l+3)},
$$$$
e_{l, m, l-1, m+1}=-\frac{\sigma \nu(l+1)}{(2 l+1)},
$$

$$
e_{l, m, l, m+1}=\frac{i \sigma \nu}{a}, \quad e_{l, m, l+1, m+1}=-\frac{\sigma \nu l}{(2 l+1)}
$$

\section{APPENDIX B: MATRIX ELEMENTS FOR THE CALCULATION OF THE INITIAL VALUES}

$$
\begin{gathered}
w_{l, m, l-1, m-1}=\frac{\sin \psi(l+m)(l+m-1)}{2(2 l+1)}, \\
w_{l, m, l+1, m-1}=-\frac{\sin \psi(l-m+2)(l-m+1)}{2(2 l+1)}, \\
w_{l, m, l-1, m}=\frac{\cos \psi(l+m)}{(2 l+1)}, \\
w_{l, m, l+1, m}=\frac{\cos \psi(l-m+1)}{(2 l+1)},
\end{gathered}
$$$$
w_{l, m, l-1, m+1}=-\frac{\sin \psi}{2(2 l+1)}, \quad w_{l, m, l+1, m+1}=\frac{\sin \psi}{2(2 l+1)},
$$$$
w_{l, m, l, m}=-\cos \psi u_{1,0}(\infty)-\sin \psi u_{1,1}(\infty) .
$$ 
*Author to whom correspondence should be addressed. Electric address: secr@ee.tcd.ie

'Deceased.

${ }^{1}$ J. L. Dormann, D. Fiorani, and E. Tronc, Adv. Chem. Phys. 98, 283 (1997).

${ }^{2}$ W. F. Brown Jr., Phys. Rev. 130, 1677 (1963).

${ }^{3}$ W. T. Coffey, J. Mol. Struct. 416, 221 (1997); Adv. Chem. Phys. 103, 259 (1998).

${ }^{4}$ A. Aharoni, Phys. Rev. 135, A447 (1964); 177, 793 (1969).

${ }^{5}$ M. Lederman, S. Schultz, and M. Ozaki, Phys. Rev. Lett. 73, 1986 (1994).

${ }^{6}$ A. Kent, S. Von Molnar, S. Gider, and D. D. Awschalom, J. Appl. Phys. 76, 6656 (1994).

${ }^{7}$ W. Wernsdorfer, B. Doudin, D. Mailly, K. Hasselbach, A. Benoit, J. Meier, J.-Ph. Ansermet, and B. Barbara, Phys. Rev. Lett. 77, 1873 (1996).

${ }^{8}$ W. Wernsdorfer, E. Bonet Orozco, K. Hasselbach, A. Benoit, B. Barbara, N. Demoncy, A. Loiseau, H. Pascard, and D. Mailly, Phys. Rev. Lett. 78, 1791 (1997).

${ }^{9}$ L. Néel, Ann. Geophys. (C.N.R.S.) 5, 99 (1949).

${ }^{10}$ W. F. Brown, Jr., IEEE Trans. Magn. 15, 1197 (1979).

${ }^{11}$ R. H. Kodama, A. E. Berkowitz, E. J. McNiff, Jr., and S. Foner, Phys. Rev. Lett. 77, 394 (1996).

${ }^{12}$ W. T. Coffey, D. S. F. Crothers, J. L. Dormann, L. J. Geoghegan, Yu. P. Kalmykov, J. T. Waldron, and A. W. Wickstead, Phys. Rev. B 52, 15951 (1995).

${ }^{13}$ W. T. Coffey, Yu. P. Kalmykov, and J. T. Waldron, The Langevin Equation (World Scientific, Singapore, 1996).

${ }^{14}$ H. A. Kramers, Physica (Amsterdam) 7, 284 (1940).

${ }^{15}$ L. J. Geoghegan, W. T. Coffey, and B. Mulligan, Adv. Chem. Phys. 100, 475 (1997).

${ }^{16}$ L. J. Geoghegan, Ph.D. Thesis, University of Dublin, 1995.

${ }^{17}$ Yu. L. Raikher and M. I. Shliomis, Adv. Chem. Phys. 87,
595 (1994).

${ }^{18}$ J. L. Dormann, F. D’Orazio, F. Lucari, E. Tronc, P. Prené, J. P. Jolivet, D. Fiorani, R. Cherkaoui, and M. Nogués, Phys. Rev. B 53, 14291 (1996).

${ }^{19}$ D. A. Smith and F. A. de Rozario, J. Magn. Magn. Mater. 3, 219 (1976).

${ }^{20}$ S. Chandrasekhar, Rev. Mod. Phys. 15, 1 (1943).

${ }^{21}$ W. T. Coffey and L. J. Geoghegan, J. Mol. Liq. 69, 53 (1996).

${ }^{22}$ I. Klik and L. Gunther, J. Stat. Phys. 60, 473 (1990).

${ }^{23}$ T. L. Gilbert, Phys. Rev. 100, 1243 (1955).

${ }^{24}$ W. T. Coffey, Yu. P. Kalmykov, and E. S. Massawe, Adv. Chem. Phys. 85, 667 (1993).

${ }^{25}$ A. R. Edmonds, Angular Momentum in Quantum Mechanics (Princeton University Press, Princeton, NJ, 1957).

${ }^{26}$ J. S. Langer, Ann. Phys. (N.Y.) 54, 258 (1969).

${ }^{27}$ P. Hänggi, P. Talkner, and M. Borkovec, Rev. Mod. Phys. 62, 251 (1990)

${ }^{28}$ I. Eisenstein and A. Aharoni, Phys. Rev. B 16, 1278 (1977); 16, 1285 (1977).

${ }^{29}$ B. J. Matowsky, Z. Schuss, and C. Tier, J. Stat. Phys. 35, 443 (1984).

${ }^{30}$ E. C. Stoner and E. P. Wohlfarth, Philos. Trans. R. Soc. London, Ser. A 240, 599 (1948).

${ }^{31}$ H. Pfeiffer, Phys. Status Solidi, 118, 295 (1990); 122, 377 (1990).

${ }^{32}$ W. T. Coffey, J. Mol. Struct. (to be published).

${ }^{33}$ W. T. Coffey, D. S. F. Crothers, Yu. P. Kalmykov, and J. T. Waldron, Phys. Rev. B 51, 15947 (1995).

${ }^{34}$ D. A. Garanin, Phys. Rev. E 54, 3250 (1996).

${ }^{35}$ Yu. P. Kalmykov, S. A. Titov, and W. T. Coffey, this issue, Phys. Rev. B 58, 3267 (1998).

${ }^{36}$ W. T. Coffey, D. S. F. Crothers, J. L. Dormann, Yu. P. Kalmykov, E. C. Kennedy, and W. Wernsdorfer, Phys. Rev. Lett. 80, 5655 (1998). 